\author{
Universidade de SÃo Paulo \\ Instituto DE FísicA
}

\title{
Estudo do resfriamento em um sistema com múltiplos estados fundamentais
}

\author{
Henrique Santos Guidi
}

Orientador:

Prof. Dr. Mário José de Oliveira

Dissertação apresentada ao Instituto de Física da Universidade de São Paulo para

obtenção do título de Mestre em Ciências

Comissão Examinadora:

Prof. Dr. Mário José de Oliveira (IFUSP)

Profa. Dra. Vera Bohomoletz Henriques (IFUSP)

Prof. Dr. Maurice de Koning (UNICAMP)

São Paulo

2007 


\section{FICHA CATALOGRÁFICA}

Preparada pelo Serviço de Biblioteca e Informação do Instituto de Física da Universidade de São Paulo

Guidi, Henrique Santos

Estudo do resfriamento em um sistema com múltiplos estados fundamentais. São Paulo, 2007.

Dissertação (Mestrado) - Universidade de São Paulo. Instituto de Física. Depto. Física Geral.

Orientador: Prof. Dr. Mario José de Oliveira

Área de Concentração: Física

Unitermos: 1. Vidro; 2. Mecânica Estatística;

3. Método de Monte Carlo 
"A vantagem de ter péssima memória é divertir-se muitas vezes com as mesmas coisas boas como se fosse a primeira vez."

Friedrich Nietzsche 


\section{Agradecimentos}

Agradeço ao professor Mário pela paciência e competência durante os vários anos de orientação. Ao professor Alberto Petri por chamar nossa atenção para o modelo estudado neste trabalho. Contribuiram para este trabalho o Maicon que sugeriu o termo gradativo, o Silas que me emprestou o seu computador por vários meses e a Carmen e a Paula que revisaram o meu texto.

Ao CNPq, pelo apoio financeiro.

Tenho uma péssima memória, então terminarei meus agradecimentos sem dar muitos nomes. A todos os meus amigos que me fazem bem e aos professores e funcionários quando fazem a universidade funcionar.

E por último, meus pais, que irei agradecer quando eu tiver a chance de continuar seu trabalho. 


\section{Resumo}

Estudamos um sistema de dois níveis acoplados como um modelo que imita o comportamento de líquidos super-resfriados que se transformam em vidros estruturais quando submetidos a um resfriamento. Em equilíbrio o modelo apresenta uma fase líquida e uma fase cristalina com diversos estados fundamentais. O modelo é definido numa rede quadrada e a cada sítio é associada uma variável estocástica de Ising. A característica que torna este modelo particularmente interessante é que ele apresenta estados metaestáveis duráveis que podem desaparecer dentro do tempo acessível para as simulações numéricas. Para imitar o processo de formação dos vidros, realizamos simulações de Monte Carlo a taxas de resfriamento constante. Apresentamos também simulações para resfriamentos súbitos a temperatura abaixo da temperatura de fusão. 


\begin{abstract}
We study a coupled two level systems as a model that imitate the behavior of supercooled liquids that become structural glasses under cooling. In the equilibrium the model shows a liquid phase and a crystalline phase with many ground states. The model is defined on a square lattice and to each site a stochastic Ising variable is associated. The feature that makes this model particularly interesting is that it displays durable metastables states which can vanish within the time available for numerical simulations. In order to imitate the glass former process, we perform Monte Carlo simulations at contanst cooling rate. We presente also simulations for quenchs to temperatures below the melting temperature.
\end{abstract}




\section{Sumário}

1 Introdução 3

1.1 Aspectos gerais . . . . . . . . . . . . . . . . . . 3

1.2 Formação . . . . . . . . . . . . . . . . . . . . 4

1.3 Paradoxo de Kauzmann . . . . . . . . . . . . . . . . . . . 6

1.4 Metaestabilidade e a relaxação temporal . . . . . . . . . . . . 6

1.5 Modelo . . . . . . . . . . . . . . . . . . . . 10

1.6 Resultados . . . . . . . . . . . . . . . . . . . 11

2 Equilíbrio termodinâmico $\quad 13$

2.1 Distribuição de Gibbs e estado fundamental . . . . . . . . . . 13

2.2 Expansão de altas temperaturas . . . . . . . . . . . . . . 15

2.3 Expansão de baixas temperaturas . . . . . . . . . . . . . . 18

2.4 Extrapolação . . . . . . . . . . . . . . . . . . . . . . . . . 19

2.5 Aproximação de campo médio . . . . . . . . . . . . . . . . 20

2.6 Método de Monte Carlo . . . . . . . . . . . . . . . . . . 23 
2.7 Propriedades termodinâmicas . . . . . . . . . . . . 25

3 Resfriamento gradativo $\quad 30$

3.1 Taxa de resfriamento . . . . . . . . . . . . . 30

3.2 Simulação . . . . . . . . . . . . . . . . . . . . . 31

3.3 Determinação do tamanho médio dos domínios cristalinos . . . 43

3.4 Regimes . . . . . . . . . . . . . . . . . 46

$\begin{array}{lll}4 & \text { Resfriamento súbito } & 49\end{array}$

4.1 Introdução . . . . . . . . . . . . . . . . . . . . . . . 49

4.2 Simulação . . . . . . . . . . . . . . . . . . . . . 50 50

$\begin{array}{llr}5 & \text { Conclusões } & 59\end{array}$ 


\section{Capítulo 1}

\section{Introdução}

\section{$1.1 \quad$ Aspectos gerais}

O objetivo de nosso trabalho é o estudo do aparecimento de estrutura vítrea nos vidros estruturais. Para isso, iremos estudar um modelo que apresenta estrutura que se assemelha àquelas dos vidros reais. Sempre que possível iremos tentar comparar nossos resultados com resultados experimentais.

Vidros são materiais amorfos com propriedades mecânicas típicas dos sólidos, muitas vezes chamados de sólidos amorfos. Os mais conhecidos são aqueles compostos principalmente de sílica, sendo comumente utilizados na produção de janelas, copos, lâmpadas, óculos, etc, por serem em geral transparentes e biologicamente inativos. Na natureza o estado vítreo é encontrado na obsidiana, uma rocha de origem vulcânica. O estado vítreo possui papel importante na tecnologia, em particular no desenvolvimento de ligas de metais amorfos [1,2]. A despeito de sua importância, há uma carência de teorias 
que o descrevam adequadamente, o que é justificado pela dificuldade de tratar sistemas de muitos corpos sem simetrias de translação. A maior dificuldade, entretanto, se deve ao fato de que os vidros não podem ser considerados sistemas em equilíbrio termodinâmico.

A estrutura revelada por difração de raio-X dos vidros de silício é bastante similar à estrutura típica dos líquidos. Por outro lado, os valores do calor específico e do coeficiente de expansão térmica são muito próximos do cristal da mesma substância [3].

\subsection{Formação}

Tipicamente, produz-se vidro aquecendo o material formador de vidro a uma temperatura maior que a temperatura de fusão $T_{m}$, e então se resfria rapidamente para temperaturas abaixo da temperatura de transição vítrea $T_{g}$. Neste processo o material passa de líquido normal para líquido super-resfriado na temperatura de fusão $T_{m}$, e passa de líquido super-resfriado a vidro na temperatura de transição vítrea $T_{g}$.

Distingue-se o líquido super-resfriado do líquido normal pelo primeiro persistir por algum tempo no estado líquido, abaixo da temperatura de fusão, ou seja, um estado metaestável comparado ao estado cristalino, que é o estado estável nessa temperatura.

Já a transição vítrea é identificada por uma queda súbita e contínua do calor específico e do coeficiente de expansão térmica, que ocorre tipicamnete a $2 / 3$ da temperatura de fusão $T_{m}$ [3]. A transformação vítrea não é uma transição de fase legítima, no sentido em que não envolve nenhum compor- 

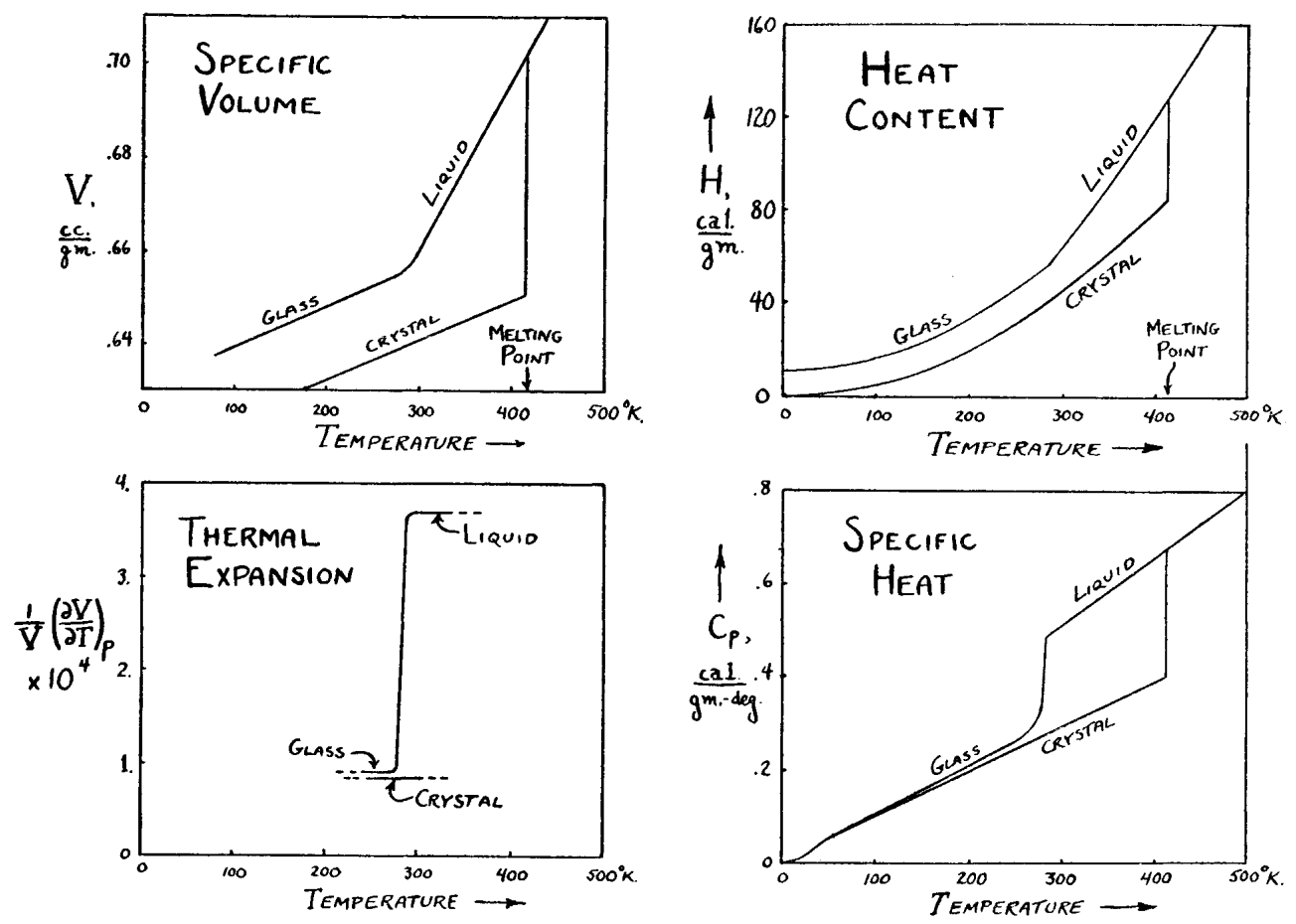

Figura 1.1: Propriedades termodinâmicas da glicose apresentadas por Kauzmann [3]

tanento singular das grandezas termodinâmicas.

Para ilustrar tal comportamento, apresentamos na figura 1.1 a reprodução da figura 1 do célebre artigo de Kauzmann [3] de 1948, onde são traçados os gráficos das propriedades termodinâmicas da glicose obtidas experimentalmente. Vemos que as grandezas apresentadas variam continuamente, contrariamente ao que ocorre na transição líquido-sólido em que as grandezas possuem uma descontinuidade. 


\subsection{Paradoxo de Kauzmann}

Ainda hoje os vidros são classificados por suas temperaturas de Kauzmann. Como a capacidade térmica do líquido é maior que a capacidade térmica do cristal, a extrapolação para temperaturas baixas do comportamento do líquido no início do super-resfriamento sugere que a entropia do líquido superresfriado tornaria-se negativa. Isso é ilustrado para o ácido lático na figura 1.2, que é a reprodução da figura 4 de Kauzmann [3].

Este fenômeno, que na verdade não acontece pois o material se torna um vidro, chama-se crise da entropia, ou paradoxo de Kauzmann[1, 3], e a temperatura na qual ocorreria é denominada de temperaura de Kauzmann $T_{K}$.

\subsection{Metaestabilidade e a relaxação temporal}

A idéia de metaestabilidade requer, por definição, que existam estados com energia livre maior que a energia livre do estado metaestável e que sejam intermediários entre as possíveis rotas no espaço de configurações entre o estado metaestável e o estado estável. Estes estados intermediários agem como barreiras pontenciais que impedem a livre passagem do estado metaestável para o estável.

Fixando os estados metaestáveis e o estado intermediário, a diferença de energia livre entre tais estados aumenta com a diminuição da temperatura. Mesmo não sendo possível formalmente descrever sistemas fora do equilíbrio por variáveis termodinâmicas como a temperatura, entropia e energia livre, 


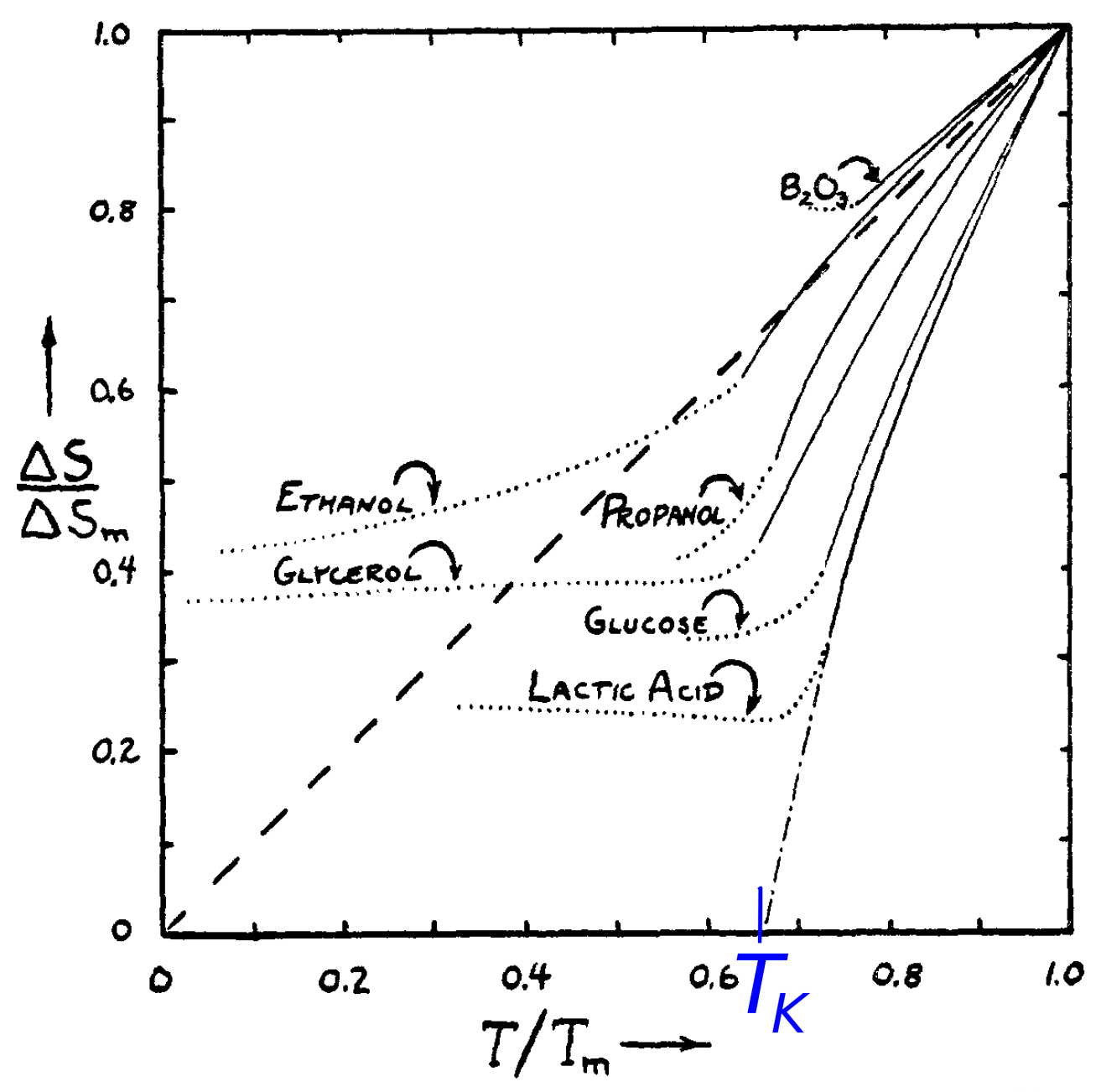

Figura 1.2: Diferença renormalizada de entropia entre o líquido superresfriado e a fase cristalina apresentados por Kauzmann [3]. 
a noção de que são as flutuações térmicas que fazem com que o sistema passe através de barreiras de energia ainda persiste.

Dessa forma, existe uma associação entre as flutuações térmicas e o tempo necessário para que o sistema passe de um estado para outro. Então, a cristalização espontânea é interpretada como o acúmulo dessas flutuações.

Entendemos que quanto mais lento for o resfriamento, maior é o tempo disponível, ou a quantidade de tentativas, para o sistema se aproximar do equilíbrio. Tal idéia é confirmada na figura 1.3, onde o estado vítreo formado pelo resfriamento mais lento tem entalpia e volume mais próximos do estado cristalino do que o vidro formado por um resfriamento mais rápido.

A temperatura de transição vítrea é definida como a temperatura tal que, abaixo dela, o tempo de relaxação de alguma grandeza física é longo comparado com o tempo de duração do experimento $t_{w}$. Logo, o valor da temperatura de transição vítrea $T_{g}$ depende essencialmente da propriedade do material a ser observada durante o experimento, a duração do experimento e o significado do termo longo.

Ilustramos de forma esquemática na figura 1.3, que é a reprodução da figura 1 de Debenedetti e Stillinger[1], a forma com que é determinada a temperatura de transição vítrea e como a taxa de resfriamento desloca o ponto em que ocorre a transição vítrea.

Ou seja, quanto mais lento for o resfriamento, maior é o tempo disponível para que o sistema se aproxime do equilíbrio, evidenciando o caracter cinético da transformação vítrea. 


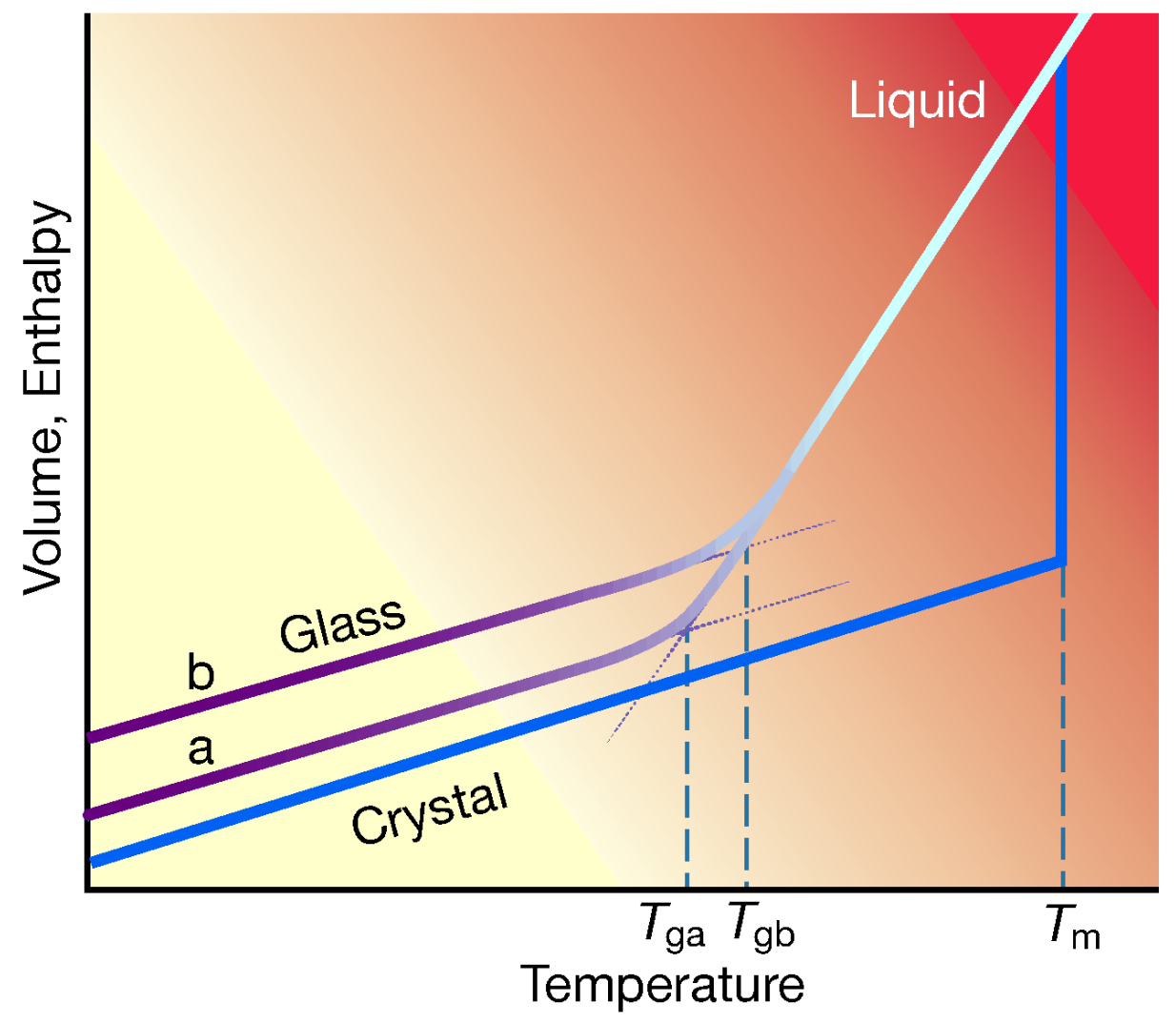

Figura 1.3: Reprodução da figura 1 de Debenedetti e Stillinger [1]. Esquema da dependência do volume e da entalpia com a temperatura a pressão constante, onde $T_{m}$ é a temperatura de fusão. As temperaturas $T_{g a}$ e $T_{g b}$ são as temperaturas de transição vítrea para resfriamentos lentos e resfriamentos rápidos respectivamente. 


\subsection{Modelo}

Muitos modelos para o surgimento da estrutura vítrea estudados na área de mecânica estatística usualmente contém ingredientes que evitam o surgimento do estado cristalino, dando margem ao aparecimento do estado vítreo. Tal é o caso dos modelos de vidro de spin [4] em que a desordem nas interações, introduzidas de forma ad hoc, induzem a formação da desordem de spin. Embora esses ingredientes sejam naturais na descrição de sistemas de spins, eles se tornam artificiais quando se trata do estudo de vidros estruturais, pois tais ingredientes suprimem a priori os possíveis estados cristalinos que são presentes em líquidos reais.

De acordo com nosso ponto de vista, as estruturas vítreas devem surgir espontaneamente em sistemas que potencialmente podem atingir estados cristalinos, reafirmando a idéia de que o estado vítreo é um estado metaestável.

O mecanismo responsável pelo aparecimento da estrutura desordenada que caracteriza o estado vítreo é a própria competição entre os diversos estados fundamentais, que deve ocorrer a baixas temperaturas. Recentemente, estudos realizados com modelos com interação de curto alcance e sem desordem a priori revelaram a possibilidade do surgimento de estados metaestáveis com vida longa obtidos após um resfriamento rápido a uma temperatura suficientemente baixa $[5,6,7,8,9,10,11,12,13]$.

Adotamos para este fim o CTLS (Coupled two levels system) introduzido por Cavagna, Giardina e Grigera [14], correspondendo a uma versão determinística de um modelo introduzido por Grigera e Israeloff [15]. 
O modelo é definido numa rede quadrada em que cada sítio possui uma variável de Ising, ou seja, cada sítio possui uma variável $\sigma_{i}$ que assume o valor +1 ou -1 . A hamiltoniana é dada por

$$
\mathcal{H}=\sum_{i}\left(1+\sigma_{i}\right) \prod_{\delta} \sigma_{i+\delta}
$$

onde a soma se estende sobre todos os sítios da rede quadrada e o produtório é entre os quatro primeiros vizinhos.

A hamiltoniana 1.1 não define por si só a dinâmica do sistema. Para o estudo dos estados metaestáveis e estados vítreos é necessário obter as propriedades do sistema como função do tempo. Para isso devemos atribuir uma dinâmica ao sistema. Escolhemos a dinâmica estocástica definida pelo algoritmo de Metropolis. Esse algoritmo é conveniente pois para tempos longos os estados gerados são os estados de equilíbrio, isto é, aqueles definidos pela distribuição de Gibbs.

\subsection{Resultados}

No próximo capítulo estudamos as propriedades de equilíbrio do sistema pela hamiltoniana 1.1, onde mostramos que o modelo apresenta o comportamento típico da transição de primeira ordem à temperatura de fusão $T_{m}$. No capítulo 3 estudamos o resfriamento gradativo, onde a cristalização é evitada com resfriamentos rápidos e a presença da fase de líquido super-resfriado e da fase vítrea são observadas. Desenvolvemos neste capítulo um método numérico para obter diretamente o tamanho médio dos domínios cristalinos $l$ e comparamos este resultado com o excesso de energia $\delta u=u-u_{\text {cristal }}$. No capítulo 4 estudamos o modelo por resfriamento súbito e observamos a 
evolução temporal do sistema e a persistência de alguns estados metaestáveis. 


\section{Capítulo 2}

\section{Equilíbrio termodinâmico}

\subsection{Distribuição de Gibbs e estado fundamen- tal}

O modelo que vamos estudar é definido numa rede quadrada em que cada sítio possui uma variável de Ising, ou seja, cada sítio possui uma variável $\sigma_{i}$ que assume o valor +1 ou -1 . A hamiltoniana é dada por

$$
\mathcal{H}=\sum_{i}\left(1+\sigma_{i}\right) \prod_{\delta} \sigma_{i+\delta}
$$

onde a soma se estende sobre todos os sítios da rede quadrada e o produtório é entre os quatro primeiros vizinhos. Usamos condições periódicas de contorno e uma rede quadrada de $N$ sitíos. Esse modelo foi introduzido por Cavagna et al [14] e corresponde a uma versão determinística de um modelo introduzido por Grigera e Israeloff [15].

Neste capítulo estudamos as propriedades do modelo no regime de e- 
quilíbrio termodinâmico. Em equilíbrio termodinâmico, a distribuição de probabilidades de um estado total do sistema $\sigma=\left(\sigma_{1}, \sigma_{2}, \ldots, \sigma_{N}\right)$ é dada pela distribuição de Gibbs

$$
P(\sigma)=\frac{1}{Z} e^{-\beta \mathcal{H}}
$$

onde $\beta=1 / T$, sendo $T$ a temperatura, e $Z$ é a função de partição

$$
Z=\sum_{\sigma} e^{-\beta \mathcal{H}}
$$

onde a soma se estende sobre todos os $2^{N}$ estados do sistema.

Um sítio que esteja no estado -1 contribui com energia zero enquanto um sítio que esteja no estado +1 contribui com energia +2 ou -2 dependendo do sinal do produto dos sinais dos vizinhos. Se o produto for negativo, a contribuição vale -2 , se for positivo, ela vale +2 . Por inspecção da Hamiltoniana, descobre-se que o estado fundamental desse modelo é obtido tomando-se uma célula unitária $5 \times 5$ tal que um quinto dos sítios esteja no estado -1 e quatro quintos no estado +1 como mostrado na figura 2.1. Essa figura mostra duas possíveis orientações da célula unitária. Como para cada possível orientação há 5 possíveis estados, obtidos por translação de um espaçamento da rede, então é possível gerar 10 estados fundamentais distintos, que denominamos estados cristalinos. Todos possuem a mesma energia por sítio

$$
u_{0}=-\frac{8}{5}
$$

Em equilíbrio termodinâmico, o modelo apresenta uma transição de fase de primeira ordem entre uma fase desordenada, que ocorre a altas temperaturas, e uma fase ordenada, que ocorre a baixas temperaturas. A transição de fase pode ser caracterizada pelo parâmetro de ordem. Olhando para o 

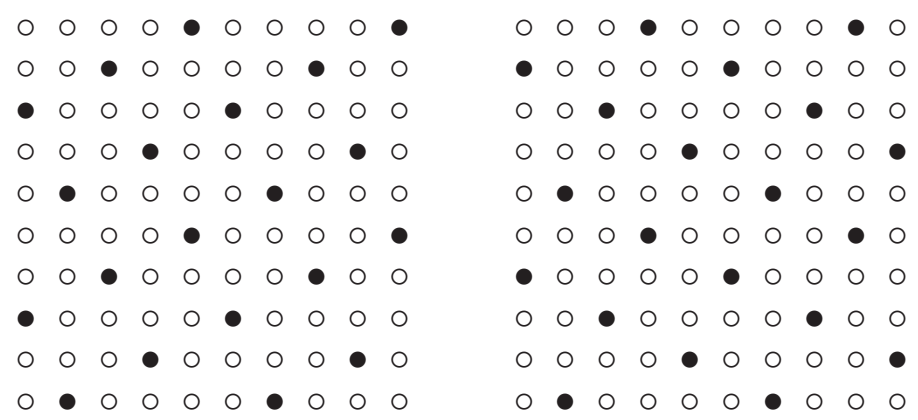

Figura 2.1: Rede cristalina com orientação Dextrogira e Levogira. Sítios de spin +1 são representados por $\circ$ e -1 por $\bullet$

estado fundamental vemos que o parâmetro de ordem deve ser definido da seguinte maneira

$$
m=\frac{1}{5}\left\{\left\langle-\sigma_{0}+\sigma_{1}+\sigma_{2}+\sigma_{3}+\sigma_{4}\right\rangle\right\}
$$

onde os sítios 1, 2, 3, e 4 são vizinhos do sitio 0 e todos pertencem a subredes distintas. No estado fundamental $m=1$. Na fase desordenada $\left\langle\sigma_{i}\right\rangle=0$ para qualquer $i$ de modo que $m=0$.

\subsection{Expansão de altas temperaturas}

Inicialmente vamos determinar as propriedades termodinâmicas da fase desordenada. Para isso recorremos a uma expansão em torno de $\beta=0[16,17]$. Começamos pela função de partição canônica que escrevemos na forma

$$
Z=2^{N} \frac{1}{2^{N}} \sum_{\sigma} e^{-\beta \mathcal{H}}=2^{N}\left\langle e^{-\beta \mathcal{H}}\right\rangle,
$$

onde aqui $\langle\ldots\rangle$ significa

$$
\langle A(\sigma)\rangle=\frac{1}{2^{N}} \sum_{\sigma} A(\sigma)
$$


Para temperaturas altas, isto é, para pequenos valores de $\beta$ podemos expandir $e^{-\beta \mathcal{H}}$ em potências de $\beta$,

$$
\left\langle e^{-\beta \mathcal{H}}\right\rangle=1-\beta\langle\mathcal{H}\rangle+\frac{\beta^{2}}{2}\left\langle\mathcal{H}^{2}\right\rangle-\frac{\beta^{3}}{6}\left\langle\mathcal{H}^{3}\right\rangle+\frac{\beta^{4}}{24}\left\langle\mathcal{H}^{4}\right\rangle+\ldots
$$

Escrevemos

$$
\mathcal{H}=\sum_{i} \mathcal{H}_{i}
$$

onde

$$
\mathcal{H}_{i}=\left(1+\sigma_{i}\right) \prod_{\delta} \sigma_{i+\delta}
$$

O valor médio de $\sigma_{i}$ é nulo

$$
\left\langle\sigma_{i}\right\rangle=0
$$

Com esse resultado, as médias das potências de $\mathcal{H}$ são calculadas a seguir.

$$
\begin{gathered}
\langle\mathcal{H}\rangle=\left\langle\sum_{i} \mathcal{H}_{i}\right\rangle=\sum_{i}\left\langle\mathcal{H}_{i}\right\rangle=\frac{1}{2^{N}} \sum_{i} \underbrace{\sum_{\{\sigma\}} \mathcal{H}_{i}}_{0}=0 \\
\left\langle\mathcal{H}^{2}\right\rangle=\left\langle\left(\sum_{i} \mathcal{H}_{i}\right)^{2}\right\rangle=\left\langle\sum_{i} \sum_{j} \mathcal{H}_{i} \mathcal{H}_{j}\right\rangle=\left\langle\sum_{i} \mathcal{H}_{i}^{2}\right\rangle+\underbrace{\left\langle\sum_{i \neq j} \mathcal{H}_{i} \mathcal{H}_{j}\right\rangle}_{0} \\
=\frac{1}{2^{N}} \sum_{i} \sum_{\sigma} 2\left(1+\sigma_{i}\right)=\frac{1}{2^{N}} \sum_{i} 2 \cdot 2^{N}=2 N
\end{gathered}
$$




$$
\begin{aligned}
& \left\langle\mathcal{H}^{3}\right\rangle=\left\langle\sum_{i} \sum_{j} \sum_{k} \mathcal{H}_{i} \mathcal{H}_{j} \mathcal{H}_{k}\right\rangle \\
& =\underbrace{\left\langle\sum_{i} \mathcal{H}_{i}^{3}\right\rangle}_{0}+\underbrace{\left\langle\sum_{i} \sum_{j \neq i} \mathcal{H}_{i} \mathcal{H}_{j}^{2}\right\rangle}_{0}+\underbrace{\left\langle\sum_{i} \sum_{j \neq i} \sum_{k \neq i, j} \mathcal{H}_{i} \mathcal{H}_{j} \mathcal{H}_{k}\right\rangle}_{0} \\
& =0 \\
& \left\langle\mathcal{H}^{4}\right\rangle=\left\langle\sum_{i} \sum_{j} \sum_{k} \sum_{l} \mathcal{H}_{i} \mathcal{H}_{j} \mathcal{H}_{k} \mathcal{H}_{l}\right\rangle \\
& =\left\langle\sum_{i} \mathcal{H}_{i}^{4}\right\rangle+\left\langle\sum_{i} \sum_{j \neq i} \mathcal{H}_{i}^{2} \mathcal{H}_{j}^{2}\right\rangle+\underbrace{\left\langle\sum_{i} \sum_{\substack{j \neq i \\
j \neq i}} \sum_{\substack{k \neq j \\
k \neq j}} \mathcal{H}_{i} \mathcal{H}_{j} \mathcal{H}_{k}^{2}\right\rangle}_{0} \\
& +\underbrace{\left\langle\sum_{i} \sum_{j \neq i} \mathcal{H}_{i} \mathcal{H}_{j}^{3}\right\rangle}_{0}+\underbrace{\left\langle\sum_{i} \sum_{j \neq i} \sum_{k \neq i, j} \sum_{l \neq i, j, k} \mathcal{H}_{i} \mathcal{H}_{j} \mathcal{H}_{k} \mathcal{H}_{l}\right\rangle}_{0} \\
& =N\left\langle\mathcal{H}_{1}^{4}\right\rangle+3 N(N-1)\left\langle\mathcal{H}_{1}^{2} \mathcal{H}_{2}^{2}\right\rangle=8 N+12 N(N-1)
\end{aligned}
$$

E com esses termos calculados obtemos a expansão para altas temperaturas, e consequentemente as funções termodinâmicas. Temos

$$
\begin{gathered}
Z=2^{N}\left\{1+\frac{\beta^{2}}{2} 2 N+\frac{\beta^{4}}{24}[8 N+12 N(N-1)]+\ldots\right\} \\
\ln Z=N\left\{\ln 2+\beta^{2}-\frac{1}{6} \beta^{4}+\ldots\right\}
\end{gathered}
$$

e portanto, a energia livre é dada por

$$
F=-\frac{1}{\beta} \ln Z=N\left\{-\frac{1}{\beta} \ln 2-\beta+\frac{1}{6} \beta^{3}+\ldots\right\},
$$

a energia média por

$$
U=-\frac{\partial}{\partial \beta} \ln Z=N\left\{-2 \beta+\frac{2}{3} \beta^{3}+\ldots\right\}
$$


e a entropia $S=(U-F) / T=\beta(U-F)$ por

$$
S=N\left\{\ln 2-\beta^{2}+\frac{1}{2} \beta^{4}+\ldots\right\}
$$

As grandezas $f=F / N, u=U / N$ e $s=S / N$ são dadas por

$$
\begin{gathered}
f=-\frac{1}{\beta} \ln 2-\beta+\frac{1}{6} \beta^{3}+\ldots \\
u=-2 \beta+\frac{2}{3} \beta^{3}+\ldots \\
s=\ln 2-\beta^{2}+\frac{1}{2} \beta^{4}+\ldots
\end{gathered}
$$

O calor específico se obtém por meio de $c=(\partial u / \partial T)$ e é dado por

$$
c=2 \beta^{2}-2 \beta^{4}
$$

O parâmetro de ordem é nulo

$$
m=0 \text {. }
$$

\subsection{Expansão de baixas temperaturas}

Também podemos fazer uma expansão a baixas temperaturas, isto é, uma expansão em torno de $T=0$. Partimos do estado fundamental e determinamos quais são os estados com energia mais próxima da energia do estado fundamental $U_{0}=-(8 / 5) N$. Os estados são aqueles obtidos do estado fundamental invertendo um sítio com sinal positivo para negativo. Nesse caso a energia aumenta de 14 unidades. O número de estados desse tipo são $(4 N / 5)$. A função de partição vale então

$$
Z=e^{-\beta N u_{0}}\left(1+\frac{4}{5} N e^{-14 \beta}\right)
$$


Logo

$$
\left.\ln Z=-\beta N u_{0}+\frac{4}{5} N e^{-14 \beta}\right)
$$

de onde obtemos

$$
\begin{gathered}
f=u_{0}-\frac{1}{\beta} \frac{4}{5} e^{-14 \beta}+\ldots \\
u=u_{0}+\frac{56}{5} e^{-14 \beta}+\ldots \\
s=\frac{4}{5}(14 \beta+1) e^{-14 \beta}+\ldots
\end{gathered}
$$

onde $u_{0}=-8 / 5$. O calor específico é dado por

$$
c=\frac{784}{5} \beta^{2} e^{-14 \beta}
$$

O parâmetro de ordem é não nulo e vale

$$
m=1-\frac{8}{25} e^{-14 \beta}
$$

Para obter a transição de fase de forma aproximada igualamos as energias livres correspondentes às fases desordenada (altas temperaturas) dada pela equação 2.27 e ordenada (baixas temperaturas) dada pela equação 2.18. Obtemos a seguinte equação

$$
-\frac{1}{\beta} \ln 2-\beta+\frac{1}{6} \beta^{3}=-\frac{8}{5}-\frac{1}{\beta} \frac{4}{5} e^{-14 \beta}
$$

cuja solução númerica é

$$
\beta=0,7576 \quad T=1,320 .
$$

\subsection{Extrapolação}

A energia interna calculada a partir da expansão de altas temperaturas acarreta uma energia livre dada pela equação 2.21. Pode-se verifica que essa 
energia livre não tem a propriedade de convexidade. Com a finalidade de obter uma energia livre com a propriedade de convexidade introduzimos ad hoc a seguinte energia interna

$$
u=-2 \tanh \beta
$$

que é igual à energia dada pelo expansão de altas temperaturas até termos cúbicos em $\beta$. A partir dela obtemos a seguinte energia livre

$$
f=-\frac{2}{\beta} \ln \cosh \beta-\frac{1}{\beta} \ln 2,
$$

que possui a propriedade de convexidade. A partir de $f$ podemos obter a entropia $s=-(\partial f / \partial T)$

$$
s=\ln 2+2 \ln \cosh \beta-2 \beta \tanh \beta .
$$

A partir de $u$ obtemos o calor específico $u=(\partial u / \partial T)$

$$
c=2 \beta^{2}\left\{1-(\tanh \beta)^{2}\right\}
$$

\subsection{Aproximação de campo médio}

Uma aproximação de campo médio $[16,17,18]$ se obtém a partir da equação exata

$$
\begin{aligned}
\left\langle\sigma_{i}\right\rangle & =\left\langle\tanh \frac{1}{2} \beta \sigma_{i} \epsilon_{i}(\sigma)\right\rangle \\
\epsilon_{i} & =\left[E\left(\sigma^{i}\right)-E(\sigma)\right],
\end{aligned}
$$

onde $E(\sigma)=\mathcal{H}(\sigma)$ é a energia da configuração $\sigma$ e $\left[E\left(\sigma^{i}\right)-E(\sigma)\right]$ é a energia da configuração obtida de $\sigma$ pela troca de sinal da variável $\sigma_{i}$. Essa equação 
será demonstrada na próxima seção. A aproximação de campo médio se obtém utilizando a aproximação

$$
\langle\tanh X(\sigma)\rangle=\tanh \langle X(\sigma)\rangle
$$

que implica em reescrever a equação 2.39 como

$$
\left\langle\sigma_{i}\right\rangle \approx \tanh \frac{1}{2} \beta\left\langle\epsilon_{i} \sigma_{i}\right\rangle
$$

Lembrando que $\epsilon_{i}$ é a diferenca de energia entre o estado $\sigma$ e o estado $\sigma^{i}$ dada por

$$
\epsilon_{i}=E\left(\sigma^{i}\right)-E(\sigma)
$$

e no nosso caso implica que

$$
\epsilon_{0}=E_{0}^{0}-E_{0}+E_{1}^{0}-E_{1}+E_{2}^{0}-E_{2}+E_{3}^{0}-E_{3}+E_{4}^{0}-E_{4} .
$$

Ou seja, a diferença de energia devido a troca do spin do sítio $i$ depende do sítio $i$, de seus primeiros vizinhos e dos primeiros vizinhos dos primeiros vizinhos, totalizando 13 sítios.

Além disso, temos $N$ equações exatas acopladas para $\left\langle\sigma_{i}\right\rangle$. Nossa primeira aproximação é reduzir o número de equações afim de representar minimamente as características do modelo. Para o nosso propósito devemos garantir a existência da estrutura cristalina, então truncamos este conjunto de equações definindo duas subredes baseadas na estrutura do cristal. A subrede $X$ para os sítios com spin -1 do cristal e a subrede $Y$ para os sítios com spin +1 do cristal. Assim temos:

$$
\begin{aligned}
& m_{X}=\left\langle\sigma_{i}\right\rangle \quad i \in X \\
& m_{Y}=\left\langle\sigma_{i}\right\rangle \quad i \in Y
\end{aligned}
$$


Vamos inicialmente ignorar as interações desacoplando os sítios, ou seja

$$
P\left(\sigma_{0}, \sigma_{1}, \sigma_{2}, \sigma_{3}, \sigma_{4}\right) \approx P\left(\sigma_{0}\right) P\left(\sigma_{1}\right) P\left(\sigma_{2}\right) P\left(\sigma_{3}\right) P\left(\sigma_{4}\right)
$$

Definimos

$$
P\left(\sigma_{i}\right)=\frac{1}{2}\left(1+m_{i} \sigma_{i}\right),
$$

que é autoconsistente com esta aproximação. Dessa definição temos convenientemente

$$
\left\langle\sigma_{i}\right\rangle=\sum_{\sigma} \sigma_{i} P(\sigma)=\sum_{\sigma} \sigma_{i} \prod_{j}^{N} P\left(\sigma_{j}\right)=\sum_{\sigma_{i}} \sigma_{i} P\left(\sigma_{i}\right)=m_{i},
$$

com

$$
\sum_{\{\sigma\}} \sigma_{0} P\left(\sigma_{0}, \sigma_{1}, \sigma_{2}, \sigma_{3}, \sigma_{4}\right)=\sum_{\sigma_{0}} \sigma_{0} P\left(\sigma_{0}\right)
$$

e as suberedes definidas, podemos calcular explicitamente $\left\langle\sigma_{0} \epsilon_{0}\right\rangle$ e $\left\langle\sigma_{1} \epsilon_{1}\right\rangle$. Temos então

$$
m_{X}=\left\langle\sigma_{0}\right\rangle \approx \tanh \frac{1}{2} \beta\left\langle\sigma_{0} \epsilon_{0}\right\rangle
$$

e

$$
m_{Y}=\left\langle\sigma_{1}\right\rangle \approx \tanh \frac{1}{2} \beta\left\langle\sigma_{1} \epsilon_{1}\right\rangle,
$$

que nos leva a duas equações acopladas para $m_{X}$ e $m_{Y}$ :

$$
\left\{\begin{array}{l}
m_{X}=-\tanh \beta\left[5 m_{Y}^{4}+4 m_{Y}^{3}\right] \\
m_{Y}=-\tanh \beta\left[5 m_{X} m_{Y}^{3}+m_{Y}^{3}+3 m_{X} m_{Y}^{2}\right]
\end{array}\right.
$$

As duas soluções triviais dadas por $m_{X}=m_{Y}=0$ a $\beta$ finito e por $m_{X}=-1$ e $m_{Y}=1$ a $\beta \rightarrow \infty$, representam o estado desordenado e o estado ordenado respectivamente. Encontramos os pontos fixos $m_{X}$ e $m_{Y}$ numericamente. As grandezas termodinâmicas são resgatadas através da Hamiltoniana resultando na seguinte expresão para a energia

$$
u=\frac{1}{5}\left(1+m_{X}\right) m_{Y}^{4}+\frac{4}{5}\left(1+m_{Y}\right) m_{X} m_{Y}^{3}
$$


e através da probabilidade (2.48) resultando na expresão para a entropia.

$$
\begin{gathered}
s=-\sum_{\sigma} P(\sigma) \ln P(\sigma) \\
s=\frac{1+m_{X}}{2} \ln \frac{1+m_{X}}{2}+\frac{1-m_{X}}{2} \ln \frac{1-m_{X}}{2}+ \\
\frac{1+m_{Y}}{2} \ln \frac{1+m_{Y}}{2}+\frac{1-m_{Y}}{2} \ln \frac{1-m_{Y}}{2}
\end{gathered}
$$

A energia livre $f$ por sítio se obtém por meio da relação $f=u-T s$.

Para a fase desordenada $m_{X}=m_{Y}=0$ resultando em

$$
u=0 \quad s=\ln 2 \quad f=-T \ln 2
$$

Para a fase ordenada os resultados são muito próximos de $m_{X}=-1$ e $m_{Y}=$ +1 , de onde obtemos os resultados

$$
u=-\frac{8}{5} \quad s=0 \quad f=-\frac{8}{5}
$$

Tais resultados indicam que a aproximação de campo médio é pouco satisfatória quando comparada com os resultados das aproximações de baixa e altas temperaturas obtidas anteriormente. O principal defeito é que a energia de ambas as fases são independentes da temperatura. Esses resultados nos conduzem a um temperatura de transição

$$
T_{m}=\frac{8}{5 \ln 2} \approx 2,3
$$

\subsection{Método de Monte Carlo}

O método de Monte Carlo [18, 19, 20] é um método numérico que gera configurações de acordo com uma distribuição de probabilidades pré determinada. 
No presente queremos gerar estados de acordo com a distribuição de Gibbs

$$
P_{n}=\frac{e^{-\beta E_{n}}}{Z}
$$

onde denotamos os estados pelo índice $n$ e a energia correspondente por $E_{n}$. O método consiste em gerar uma cadeia de Markov tal que a probabilidade estacionária seja a distribuição de Gibbs acima. A cadeia de Markov é definida pela probabilidade de transição de um dado estado $n$ para um outro estado $m$, que denotamos por $p(n \mid m)$, que é também a probabilidade condicional do estado $n$ dado o estado $m$.

A implementação do método de Monte Carlo pode ser feita através de diversas variantes conhecidas como algoritmos. Descrevemos abaixo o algoritmo de Metropolis cuja probabilidade de transição é definida por

$$
p(n \mid m)=\min \left\{1, e^{-\beta\left(E_{n}-E_{m}\right)}\right\}
$$

Para o modelo que estudamos bem como para modelos como o modelo de Ising, o algoritmo de Metropolis é:

- Escolha um sítio da rede.

- Calcule a varição $\Delta E$ de energia se for alterado o estado do sítio.

- Se a energia diminuir o novo estado do sítio é aceito, se não, o novo estado é aceito com probabilidade $e^{-\beta \Delta E}$.

Cada vez que este procedimento é feito para todos os sítios da rede é contado um passo de Monte Carlo pmc. O tempo em nossas simulações é medido em passos de Monte Carlo. 
Através de algoritmo geramos um número de configurações e a partir delas estimamos as grandezas termodinâmicas. Por exemplo a energia média é estimada por

$$
\bar{E}=\frac{1}{M-N} \sum_{n=N+1}^{M} E_{n},
$$

onde $M$ é o número total de configurações geradas e $N$ é o número de configurações iniciais descartadas.

Um outro algoritmo muito utilizado é o de Glauber. A probabilidade de transição desse algoritmo é dada por

$$
p(n \mid m)=\frac{1}{2}\left\{1-\tanh \left(\frac{1}{2} \beta\left[E_{n}-E_{m}\right]\right)\right\}
$$

Entretanto, não utilizamos esse algoritmo nas simulações. Todas as simulações foram feitas utilizando o algoritmo de Metropolis.

\subsection{Propriedades termodinâmicas}

Na figura 2.2 apresentamos a energia média por sítio $u$ determinado pelo método de Monte Carlo para uma rede de $N=50 \times 50$. Para comparação mostramos também as aproximações analíticas dadas pelas equações 2.35 e 2.22. O modelo apresenta uma transição de fase descontínua na temperatura $T_{m}$. Calculamos a temperatura de transição a partir do gráfico da energia livre de Helmholtz, mostrado na figura 2.3, que é determinada por integração de $(\partial \beta f / \partial \beta)=u$.

$$
\beta f=\beta_{0} f\left(\beta_{0}\right)+\int_{\beta_{0}}^{\beta} u\left(\beta^{\prime}\right) d \beta^{\prime}
$$




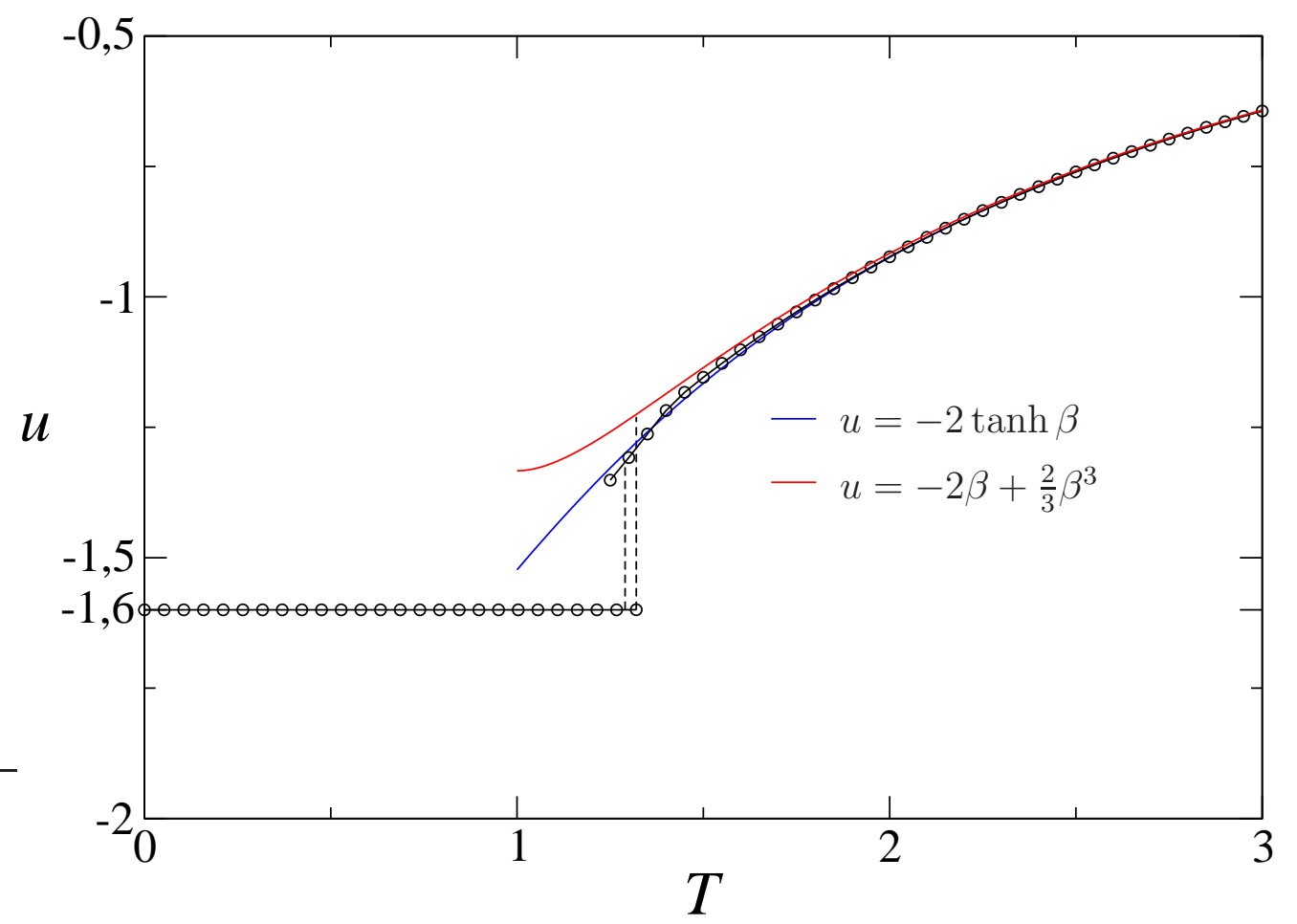

Figura 2.2: Energia por sitio $u$ determinado pelo método de Monte Carlo (círculos) e pelas aproximações analíticas de altas temperaturas em vermelho e a sua extrapolação em azul dadas pelas equações 2.35 e 2.22. As linhas tracejadas indicam o ponto em que ocorre a transição pelos resultados da simulação $T_{m}=1,29$ e pela aproximação de altas temperaturas $T_{m}=1,32$.

A constante $\beta_{0} f\left(\beta_{0}\right)$ é obtida comparando o resultado numérico com a aproximação analítica válida para altas temperaturas.

Para baixas temperaturas, na fase cristalina, a energia é praticamente constante e igual à energia do estado fundamental, $u=-1,6$. Utilizando esse resultado, a energia livre da fase cristalina vale $f=-1,6$. A transição ocorre no ponto em que as energias livres do líquido e do cristal se igualam. A partir dos gráfico 2.3 estimamos o seguinte valor: $T_{m}=1,29(1)$. 


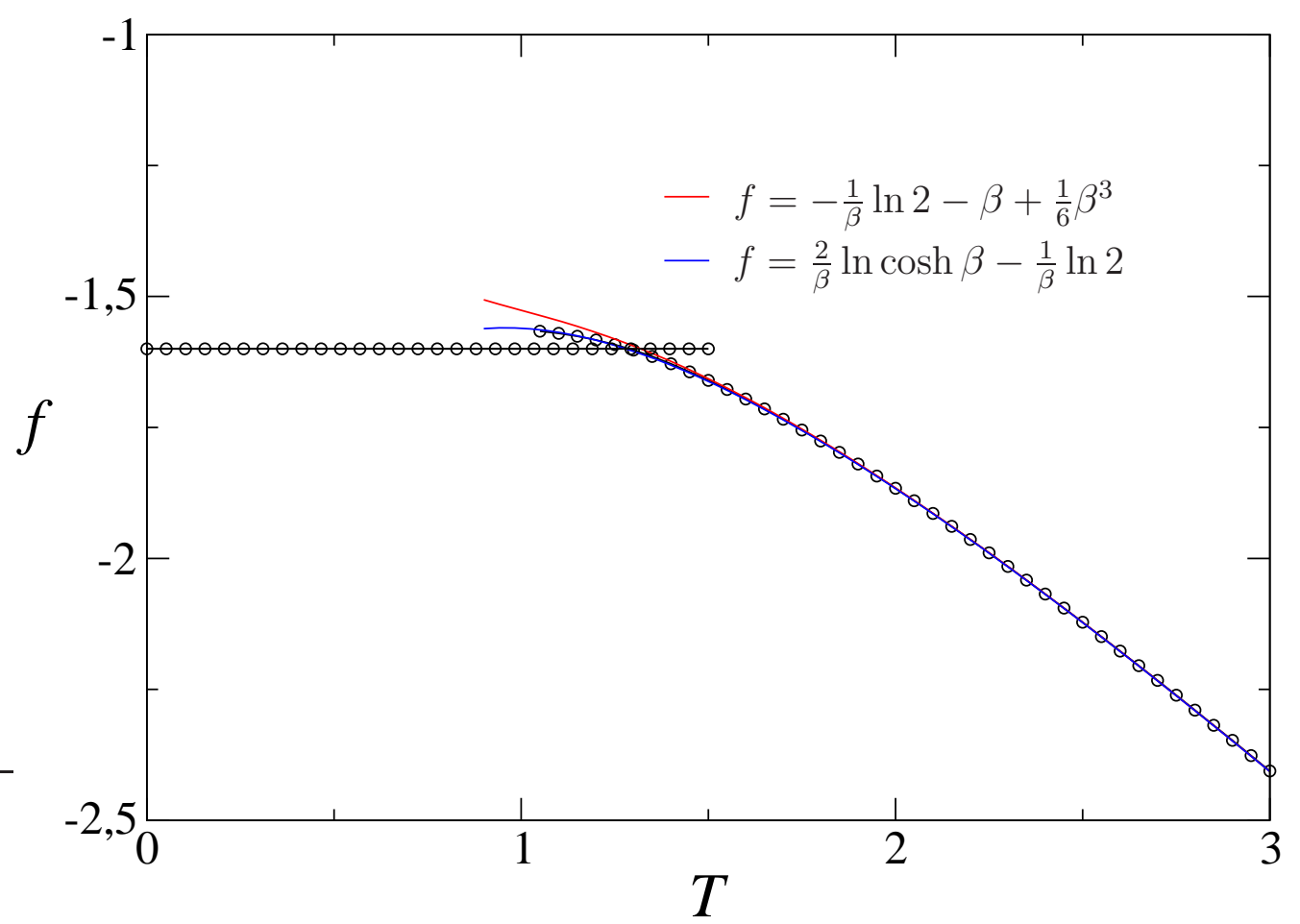

Figura 2.3: Energia livre de Helmholtz por sitio $f$ determinada por integração dos resultados das simulações feitas pelo método de Monte Carlo (círculos) e pelas aproximações analíticas de altas temperaturas em vermelho e a sua extrapolação em azul dadas pelas equações 2.36 e 2.21. Pelo resultado da simulação, estimamos que a transição de fase ocorre a $T_{m}=1,29$ e pela aproximação de altas temperaturas a $T_{m}=1,32$. 


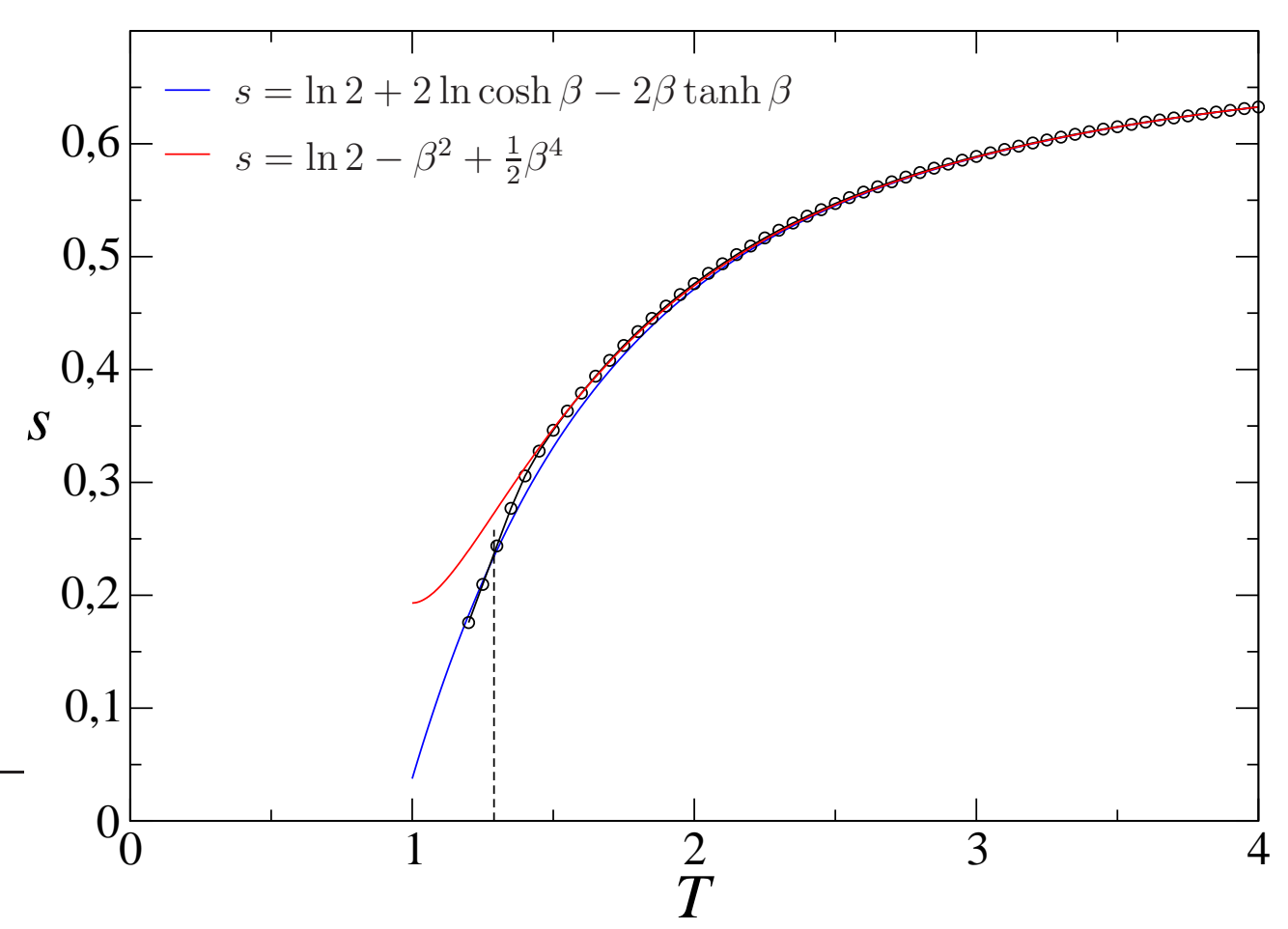

Figura 2.4: Entropia por sitio $s$ determinada pelo método de Monte Carlo (círculos) e pelas aproximações analíticas de altas temperaturas em vermelho e a sua extrapolação em azul dadas pelas equações 2.23 e 2.37. A linha tracejada indica o ponto em que ocorre a transição pelos resultados da simulação $T_{m}=1,29$. 


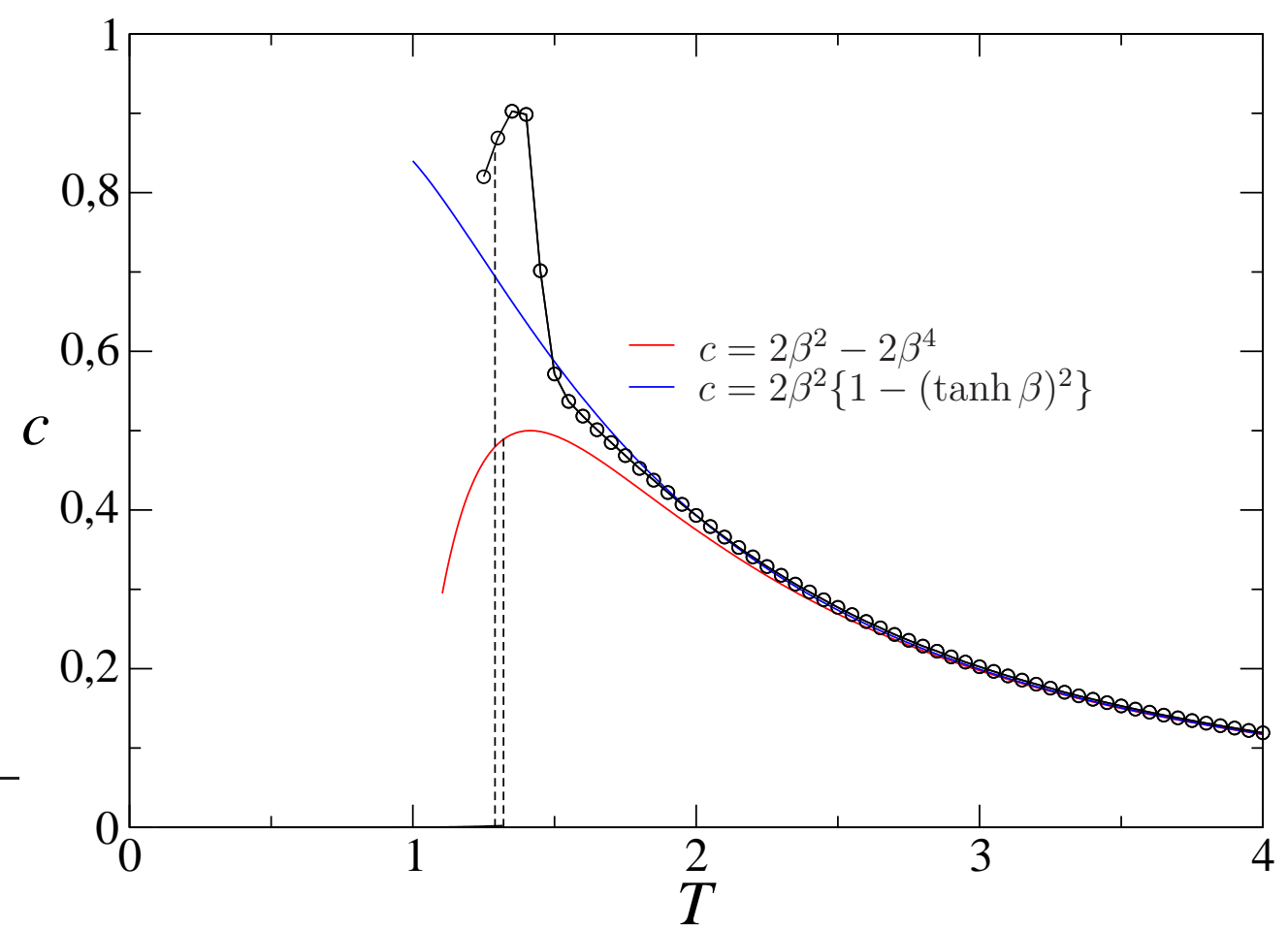

Figura 2.5: Calor específico por sitio $c$ determinado por integração dos resultados da simulação (círculos) e pelas aproximações analíticas de altas temperaturas em azul e a sua extrapolação em vermelho dadas pelas equações 2.24 e 2.38. As linhas tracejadas indicam o ponto em que ocorre a transição pelos resultados da simulação $T_{m}=1,29$ e pela aproximação de altas temperaturas $T_{m}=1,32$. 


\section{Capítulo 3}

\section{Resfriamento gradativo}

\subsection{Taxa de resfriamento}

Neste capítulo tentaremos comparar o comportamento do modelo CTLS (Coupled two levels system) com o comportamento dos vidros reais. Em particular, com os resultados de Kauzmann [3].

A transição vítrea é um fenômeno cinético que ocorre quando o sistema é resfriado. Tipicamente se prepara o material formador do vidro a uma certa temperatura acima da temperatura de transição liquido-cristal e então o sistema é resfriado, por exemplo, a uma velocidade de resfriamento constante $r$

$$
r=\frac{d T}{d t}
$$

Uma grandeza relevante para caracterizar este processo é a energia em excesso $\delta U$ e o comprimento característico do sistema no final do processo de resfriamento a temperatura nula $l_{0}$, que pode ser encarado como o ta- 
manho médio dos domínios cristalinos [5]. Quanto mais lento for o resfriamento maior será o comprimento característico pois o sistema tem mais tempo, mais tentativas, para encontrar o caminho até algum mínimo local mais abrangente.

Fenomenologicamente espera-se que o crescimento dos domínios em vídros seja muito lento. Mais precisamente, espera-se que os domínios em vidros cresçam apenas logaritmamente com o inverso da taxa de resfriamento [5].

$$
l_{0} \sim \ln \frac{1}{r}
$$

\subsection{Simulação}

Tendo em vista que a transição para o estado vítreo é um fenômeno cinético, devemos dotar o modelo definido pela hamiltonina 2.1 de uma dinâmica que represente a sua evolução temporal durante o resfriamento. Escolhemos a dinâmica estocástica definida pelo método de Monte Carlo. Utilizamos, nesse estudo, o algoritmo de Metropolis, como explicado no capítulo anterior. Essa dinâmica simula o contato do sistema com um banho térmico à temperatura $T$.

Preparamos o sistema para nossas simulações com um estado totalmente desordenado, isto é, a cada sítio da rede atribuímos o valor +1 ou -1 com probabilidades iguais. Em seguida, executamos 100 passos de Monte Carlo pmc a uma temperatura $T_{0}$ muito maior do que a temperatura de transição $T_{m}$. Observamos que para essa temperatura $T_{0}$ o sistema alcança o equilíbrio muito rapidamente. 
O resfriamente é feito o mais contínuo possível a partir da última configuração obtida com a temperatura $T_{0}$. A taxa de resfriamento $r=d T / d t$ é feita constante de modo que a temperatura do banho térmico varia com o tempo de acordo com

$$
T=T_{0}-r t
$$

onde $t$, o tempo, é medido em $p m c$, isto é, a unidade de tempo é discreta e igual a um pmc. A temperatura do banho térmico é modificada de um incremento $\Delta T$ a cada passo de Monte Carlo $\Delta t$ de modo que $\Delta T=r \Delta t$. Para taxas de resfriamento altas partimos de $T_{0}=10$, para taxas de resfriamento baixas usamos $T_{0}=5$, conforme se vê na tabela 3.1. As estimativas das grandezas relevantes foram obtidas por médias de várias realizações. Cada realização corresponde a uma simulação com os mesmos parâmetros mas com uma sequência de números aleatórios diferentes. O número de realizações está apresentada na tabela 3.1. Todas as simulações foram realizadas em redes quadradas de largura $L=50$ com condições periódicas de contorno. Utilizamos o gerador de números aleatórios denominado ranlux389 [21, 22] e o próprio gerador padrão do compilador gcc[19, 23].

Na figura 3.1 apresentamos um exemplo de configuração do modelo à temperatura de $T=0,25$, abaixo da temperatura de transição, obtida por resfriamento a uma taxa $r=0,00002$. A configuração mostrada apresenta regiões ordenadas localmente de acordo com os vários estados fundamentais e deve ser entendida como um estado metaestável já que a essa temperatura a configuração de equilíbrio é aquela correspondente ao estado cristalino.

Na figura 3.2 apresentamos o gráfico da energia por spin para diversas taxas de resfriamento. Observamos que para taxas de resfriamento menores o sistema se aproxima cada vez mais do estado ordenado. Neste gráfico vemos 


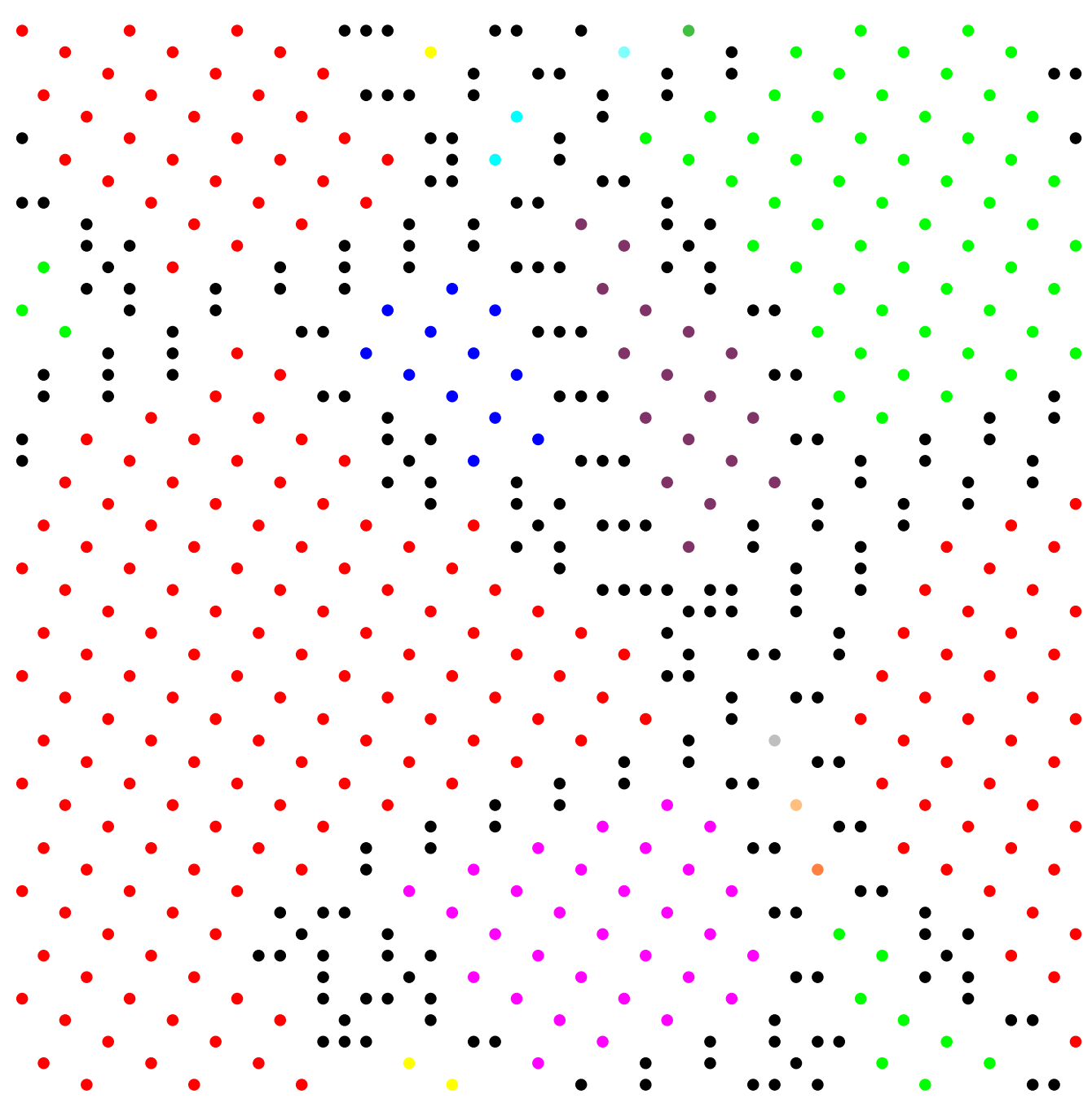

Figura 3.1: Exemplo de configuração a temperatura de banho térmico nula $T=0$ para uma rede quadrada de $50 \times 50$ sítios com condições periódicas de contorno após resfriamento a taxa constante $r=0,0000032$. São apresentados apenas os sítios de spin -1. A configuração mostrada apresenta os sítios pertecentes à regiões desordenadas em preto e os sítios pertecentes às regiões ordenadas de acordo com os vários estados fundamentais em cores, afim de discriminar os diferentes domínios cristalinos. 
Tabela 3.1: Tabela das taxas de resfriamento $r$, número de realizações $R$ e temperatura inicial de resfriamento $T_{0}$.

\begin{tabular}{|l|r|r|}
\hline$r$ & $R$ & $T_{0}$ \\
\hline 0,05 & 4843 & 10 \\
0,01 & 3001 & 10 \\
0,002 & 1274 & 10 \\
0,0004 & 497 & 10 \\
0,00008 & 1210 & 5 \\
0,000016 & 883 & 5 \\
0,0000032 & 363 & 5 \\
0,00000064 & 197 & 5 \\
\hline
\end{tabular}

que o sistema permanece estacionado em patamares de energia abaixo de uma certa temperatura característica $T_{g}$. Identificamos essa temperatura como a temperatura de transição vítrea, ou seja, a temperatura tal que, abaixo dela, o tempo de relaxação é longo comparado à duração do experimento [3]. De fato, os tempos característicos crescem tanto que não se observa sinais claros de relaxamento no período $T_{g} / r$ entre a ocorrência da transição vítrea e o fim do experimento.

A determinação de $T_{g}$ é feita como em [1,24], e está ilustrada no gráfico da figura 3.3. A temperatura $T_{g}$ é definida pelo cruzamento da reta tangente à curva a temperatura nula e a reta tangente ao ponto da curva de maior coeficiente angular, ou seja, o ponto de inflexão.

Por se tratar de um resfriamento, que não é suficientemente lento para garantir o equilíbrio térmico entre o sistema e o banho térmico, a temperatura efetiva do sistema é sempre maior do que a temperatura do banho térmico. 


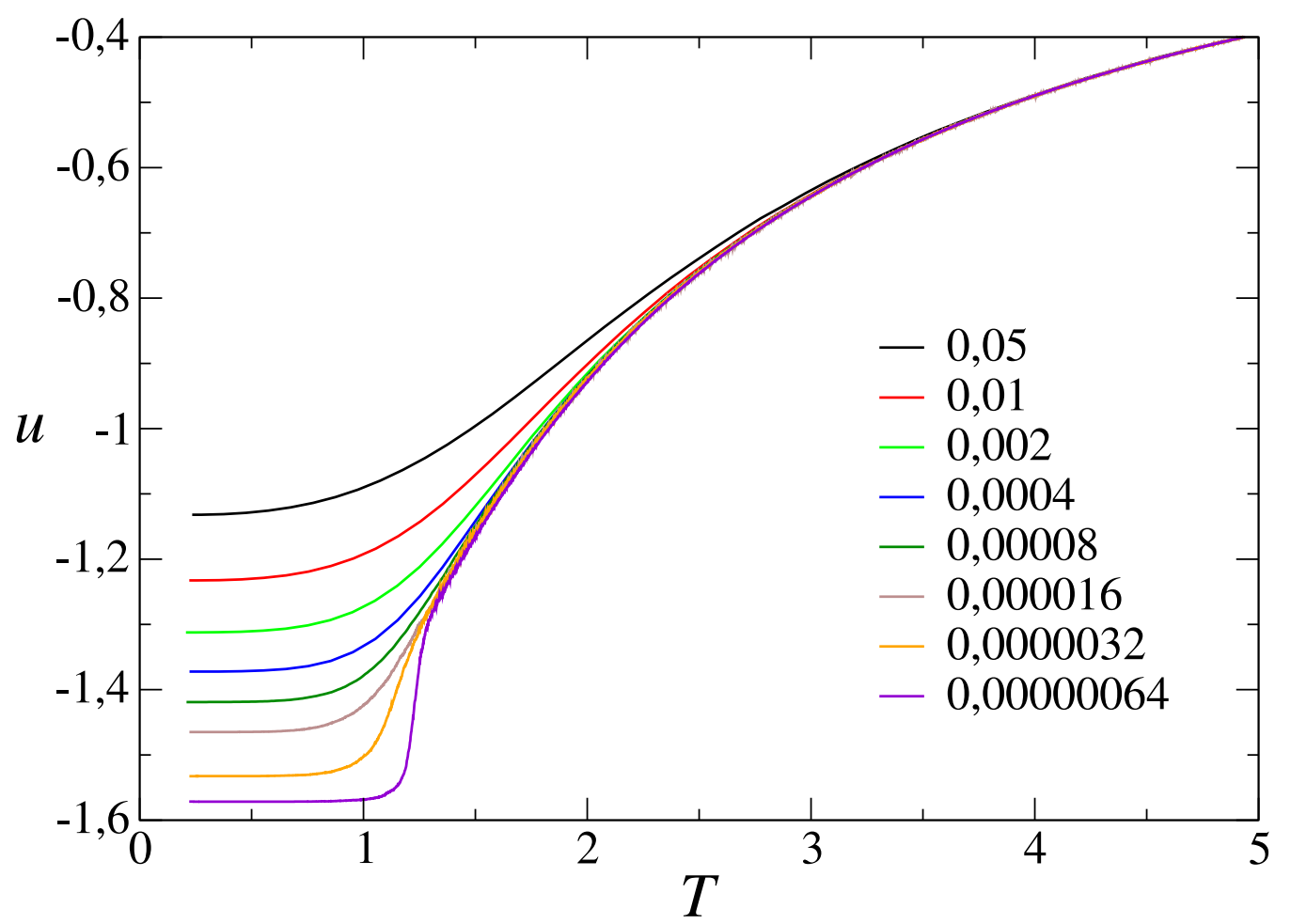

Figura 3.2: Energia por sítio $u$ versus temperatura $T$ para diversas taxas de resfriamento $r$. Este gráfico deve ser comparado com o gráfico da figura 1.3, onde confirmamos que o modelo apresenta o comportamento típico dos vidros. Observa-se que o sistema baixo da temperatura de transição $T_{m}$ aproxima-se do estado fundamental a $u_{\text {cristal }}=-1,6$ com resfriamentos mais lentos. Devemos salientar que por ser um modelo definido em um reticulado, a noção de volume e presão não se aplicam e por isso a entalpia e a energia nesse modelo são equivalentes. 


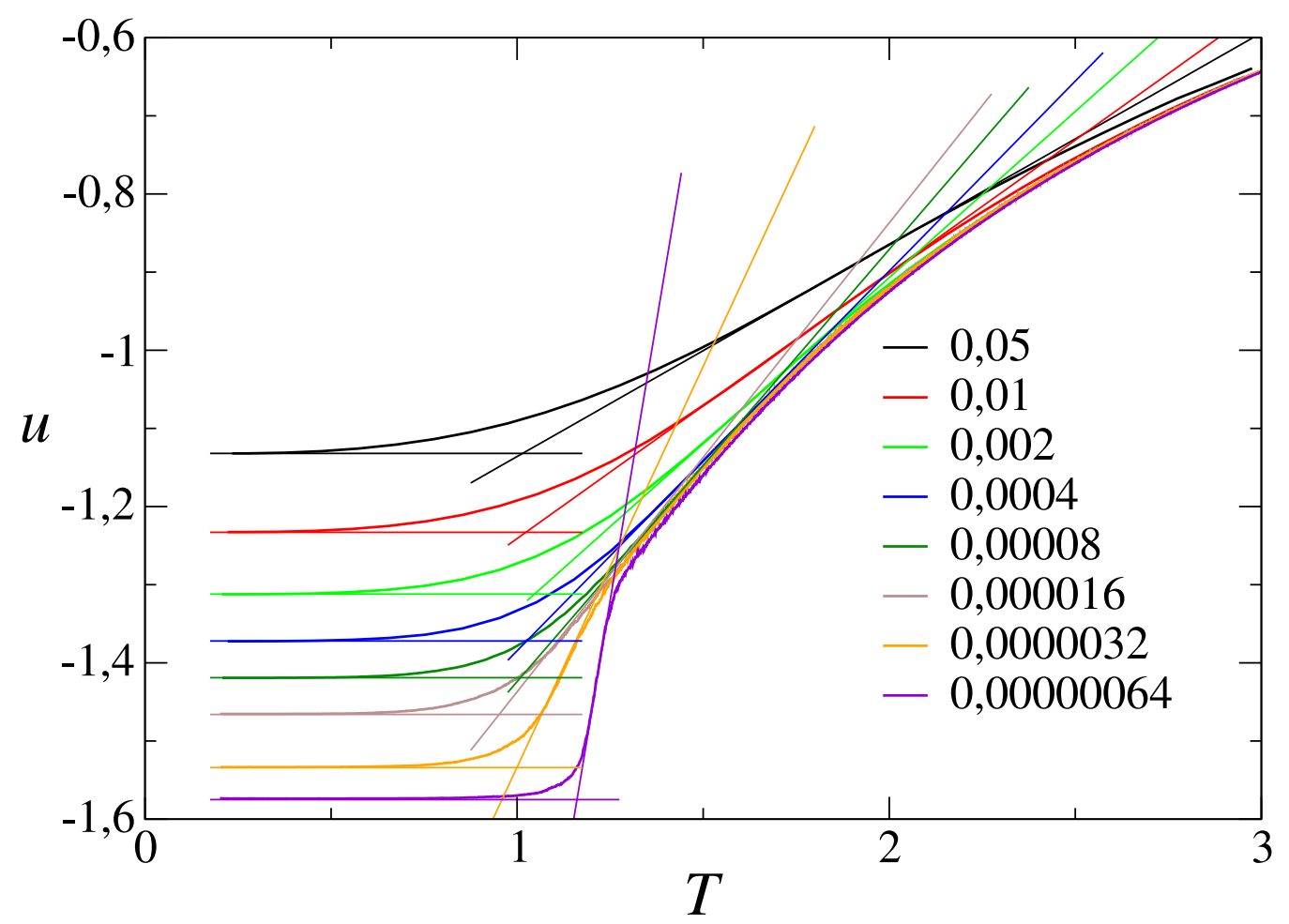

Figura 3.3: Energia por sítio $u$ versus temperatura $T$ para diversas taxas de resfriamento $r$. As retas inclinadas são tangentes ao ponto de inflexão das curvas correspondentes. As retas horizontais indicam a menor energia atingida em cada resfriamento. A temperatura $T_{g}$ corresponde ao ponto de cruzamento dessas retas. 
Este atraso aumenta com o crescimento da taxa de resfriamento, ou seja, quanto maior a taxa de resfriamento maior a diferença entre a temperatura real do sistema e a temperatura do banho térmico.

Apesar das relações termodinâmicas não estarem definidas fora do equilíbrio termodinâmico, vamos utilizá-las afim de determinar as propriedades termodinâmicas para cada uma das taxas de resfriamento. Para isso adotamos a temperatura do sistema como sendo aquela do banho térmico. A partir da energia por sítio $u$ do sistema dada pelo gráfico 3.2 determinamos a energia livre por sítio $f$, a entropia por sítio $s$ e o calor específico $c$ da mesma maneira como fizemos no capítulo anterior. Essas grandezas como função da temperatura são mostradas nos gráficos das figuras 3.4, 3.5 e 3.6. Na figura 3.4 é apresentado o gráfico da energia livre de Helmholtz como função da temperatura. Vemos que quanto maior a taxa de resfriamento, maior a energia livre do estado super-resfriado.

Experimentalmente, a temperatura de transição vítrea $T_{g}$ cresce com a taxa de resfriamento $r$ [1]. Os valores da temperatura de transição vítrea $T_{g}$ obtidos para cada taxa de resfriamento são apresentados na figura 3.7 juntamente com a temperatura de Kauzmann $T_{K}$. Observamos que para o CTLS a temperatura de transição vítrea $T_{g}$ é quase uma constante próxima a $T=1,0$ e decresce apenas com as taxas de resfriamento $r$ bem baixas, como se fosse empurrado pela temperatura de Kauzman $T_{K}$, que é sempre menor que a temperatura de transição vítrea $T_{g}$. Dessa forma, contrariando o esquema baseado em observações experimentais apresentado na figura 1.3. Vemos tanto neste esquema como no gráfico do volume específico da glicose da figura 1.1 que a região da curva do resfriamento referente ao líquido superresfriado é praticamente uma reta, muito diferente das curvas para a energia 


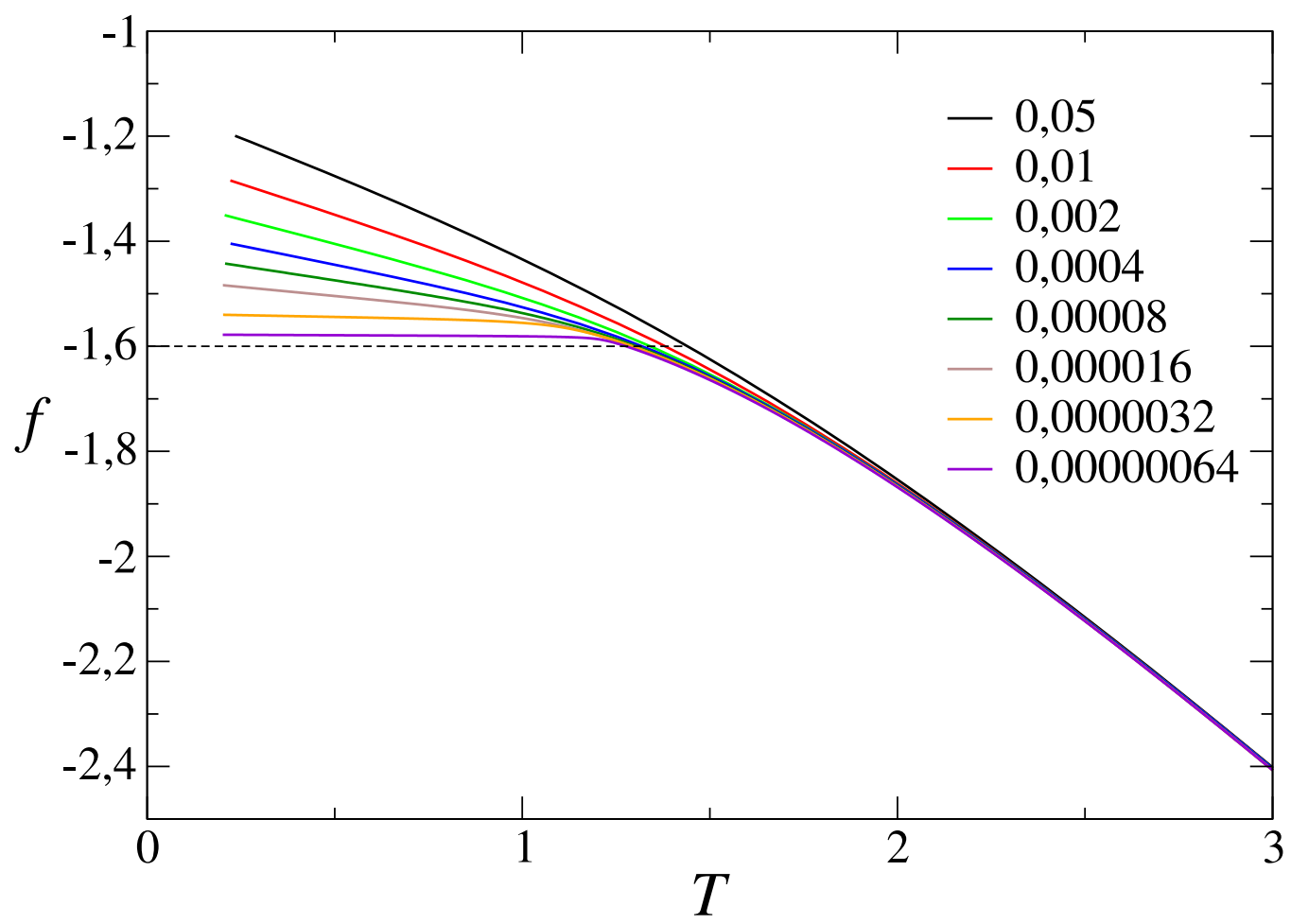

Figura 3.4: Gráfico da energia livre de Helmholtz para várias taxas de resfriamento $r$ (para comparação mostramos a energia do cristal (linha tracejada)). É importante notar que a linha tracejada do cristal é praticamente uma reta horizontal a $f=-1,6$. 


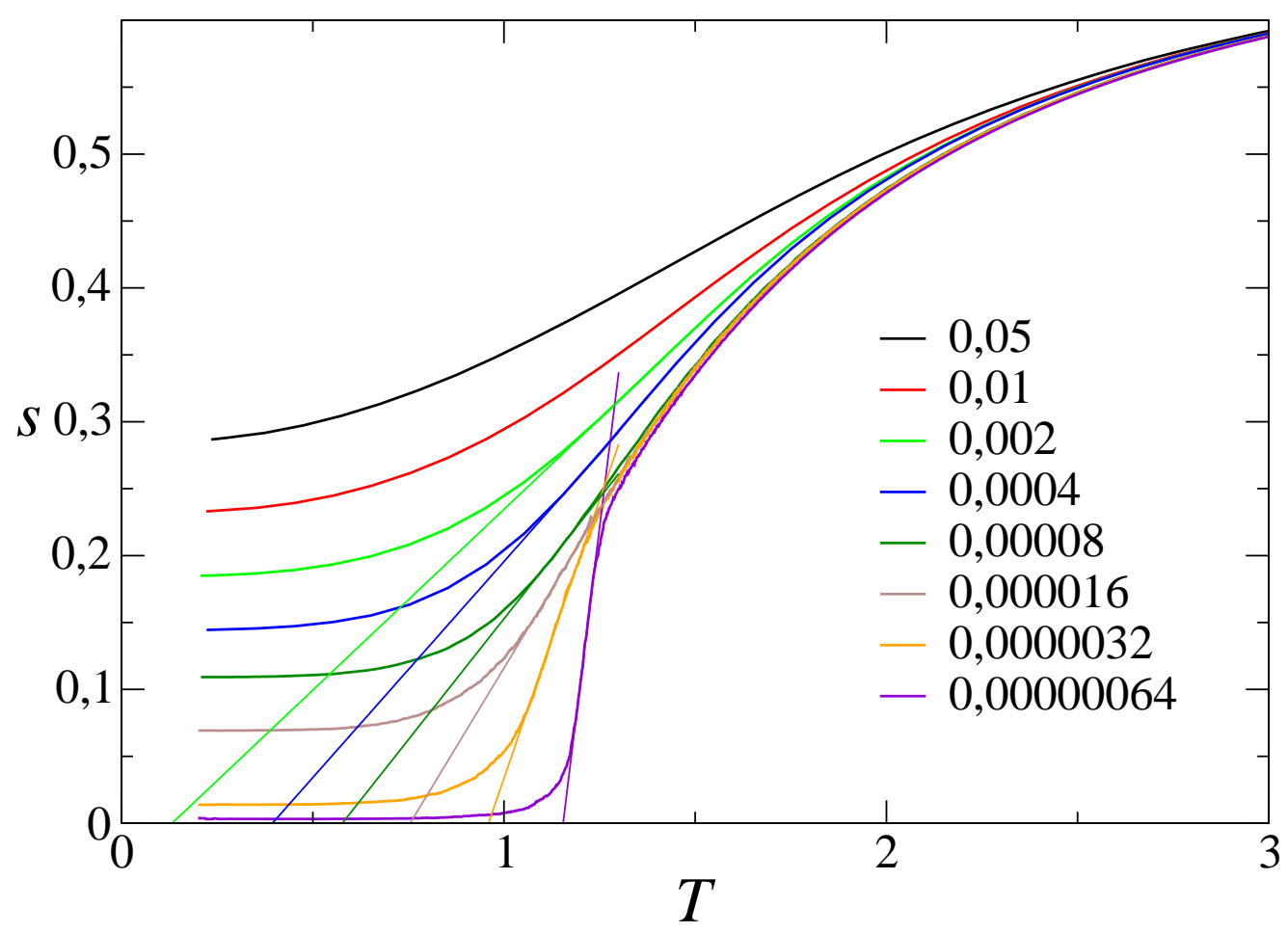

Figura 3.5: Gráfico da entropia por spin pela temperatura. As retas inclinadas são tangentes ao ponto de inflexão das curvas correspondentes como uma extrapolação para baixas temperaturas do líquido super-resfriado. Obtemos assim $T_{K}$, a temperatura tal que a extrapolação do líquido super-resfriado tem entropia igual à entropia do cristal. 


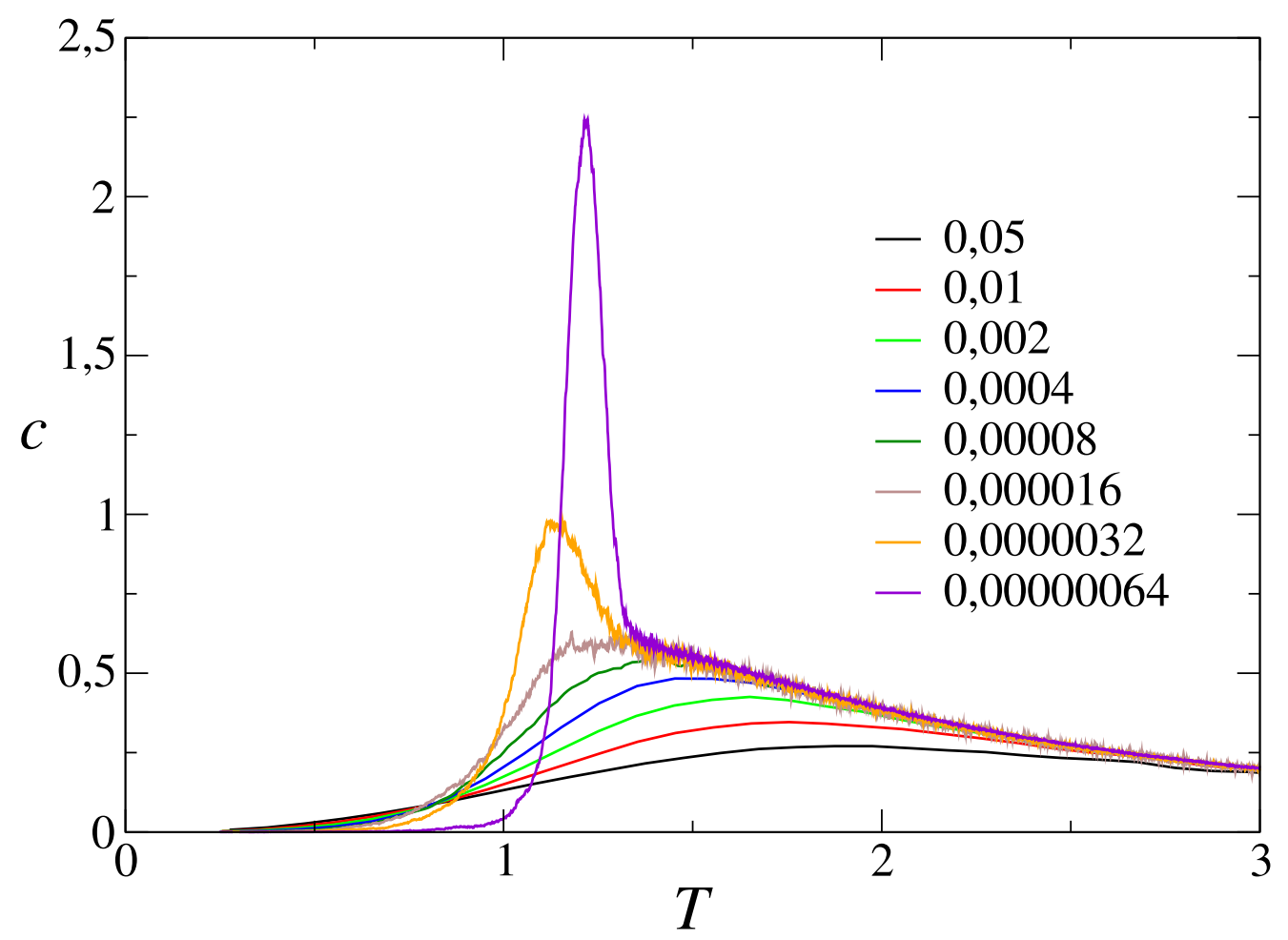

Figura 3.6: Gráfico do Calor Específico pela temperatura. 
Tabela 3.2: Tabela das taxas de resfriamento $r$, temperatura de Kauzmann $T_{K}$, temperatura de transição vítrea $T_{g}$ e temperatura de transição líquidocristal $T_{m}$.

\begin{tabular}{|l|l|r|}
\hline$r$ & $T_{K}$ & $T_{g}$ \\
\hline 0,05 & & 1,01 \\
0,01 & & 1,02 \\
0,002 & 0,13 & 1,04 \\
0,0004 & 0,39 & 1,02 \\
0,00008 & 0,58 & 1,01 \\
0,000016 & 0,75 & 0,95 \\
0,0000032 & 0,97 & 1,00 \\
0,00000064 & 1,15 & 1,16 \\
\hline
\end{tabular}

por sítio obtida em nossas simulações. A entropia do líquido na temperatura de liquefação é maior que a entropia do cristal correspondente. Devida a capacidade térmica do líquido ser maior que a do cristal, a diferença entre as entropias decresce com o super-resfriamento[3].

Para ácidos láticos esta diferença entre entropias decresce tão rápido que a extrapolação de dados experimentais contemporâneos a Kauzmann prevê o paradoxo de Kauzmann e a entropia do líquido iria para valores negativos para temperaturas menores que $T_{K}$, o que não pode acontecer já que a entropia é uma grandeza inerentemente positiva [1]. Na prática, a transição vítrea interfere e o paradoxo não acontece.

Como a energia do sistema cresce com a temperatura, espera-se que a energia seja algo parecido com o inverso do grau de cristalização do sistema $[5]$. 


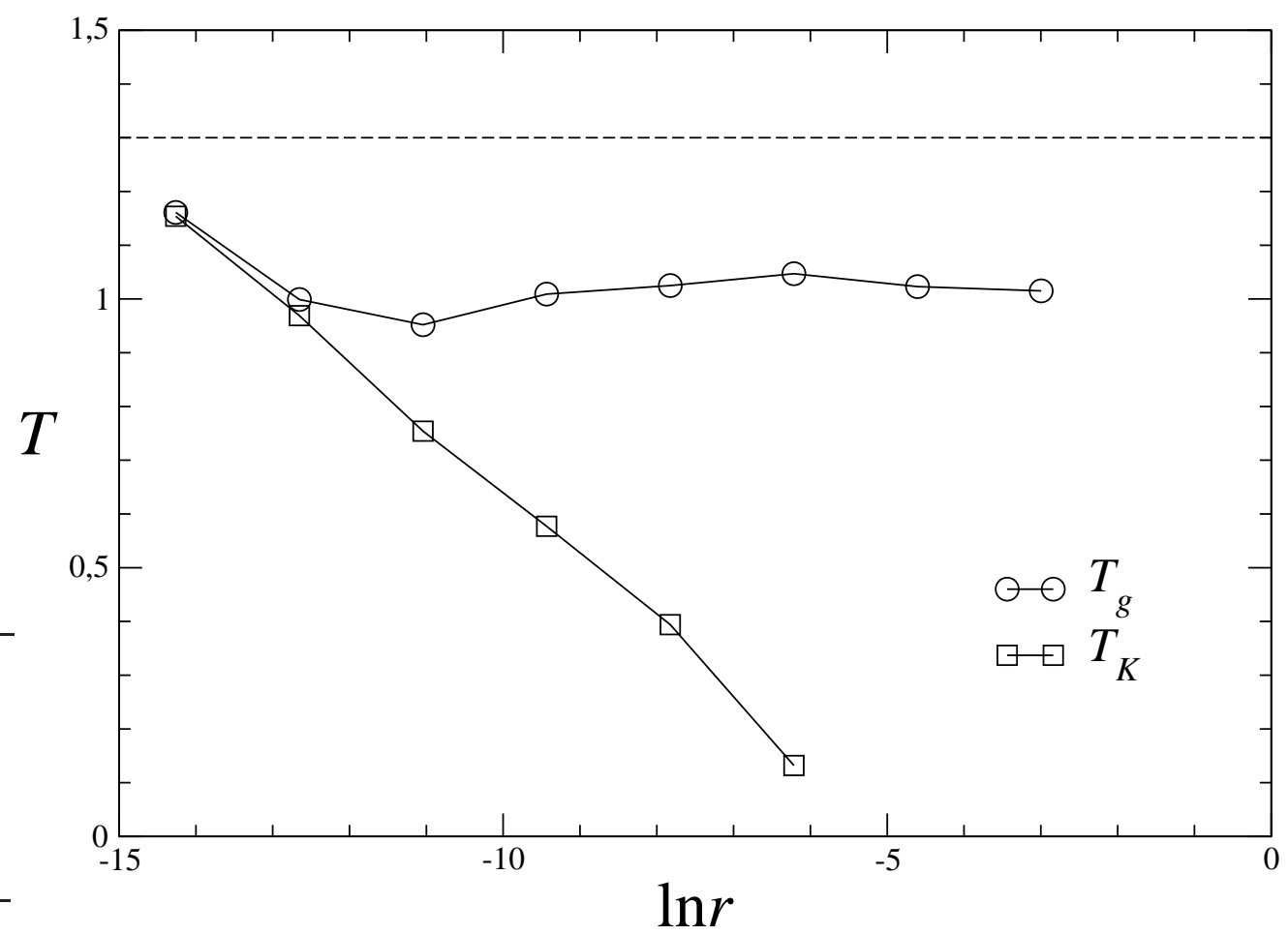

Figura 3.7: Gráfico da temperatura de transição vítrea $T_{g}$ e da temperatura de Kauzmman $T_{K}$ pelo logaritmo da velocidade de resfriamento $r$. Como referência mostramos a temperatura de transição vítrea $T_{m}=1,30$ (linha tracejada). 


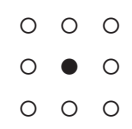

Figura 3.8: Spin cristalizado.

$$
\delta U \sim \frac{1}{l_{0}}
$$

Para conferir esta relação bastante razoável, precisamos medir o tamanho típico dos domínios cristalinos.

\subsection{Determinação do tamanho médio dos do- mínios cristalinos}

As células unitárias, representadas na figura 2.1, são relativamente grandes, o que dificulta a contagem de sítios pertencentes a cristais. Além disso, estas células são insensíveis à blocos de spins no estado fundamental menores que $5 \times 5$. Então optou-se por abandonar as células unitárias como referência a configuração cristalina e adotou-se o spin cristalizado [14], que pode também ser interpretado como a representação no estado fundamental do parâmetro de ordem dado pela equação 2.5 .

O spin cristalizado é definido como o sítio tal que seu spin vale -1 e está cercado por 8 sítios, compartilhados com outro spin cristalizado, de spin +1 . A figura 3.8 ilustra tal configuração.

A configuração de 9 spins da figura 3.8 é suficientemente pequena para distinguir blocos $3 \times 3$, e suficientemente grande para ter baixa ocorrência a 
temperatura altas, mas não o suficiente para ser sensível à orientação.

Domínios cristalinos são um conjunto de spins cristalizados posicionados como na figura 2.1. São ditos levógiros se são simétricos por deslocamentos de $\pm(1,2)$ e $\pm(2,-1)$ e dextrógiros se são simétricos por deslocamentos de $\pm(1,-2)$ e $\pm(2,1)$.

Por simplicidade, vamos ignorar a diferença entre as duas possíveis orientações e identificar a ocorrência dessa relação espacial entre spins cristalizados como uma ligação entre 2 spins cristalizados. Assim, pares de spins cristalizados com esta ligação pertecem ao mesmo domínio cristalino. Criamos dessa forma um critério para identificar os domínios e determinar seu tamanho médio.

Como no cristal alguns sítios do spin cristalizado são compartilhados com outros spins cristalizados, os oitos primeiros vizinhos tem peso meio e por isso a massa de cada spin cristalizado é 5 .

Para cada sítio da rede associamos a variável de spin $\sigma$, e para identificar os sítios que pertencem ao mesmo domínio cristalino atribuimos a cada sítio a variável $i d$. Isto permite a enumeração dos domínios cristalinos.

Para a determinação da quantidade de domínios $n$, desenvolvemos um algoritmo recursivo, que é um método comum de simplificação consistindo em dividir um problema em subproblemas semelhantes. A nossa implementação é dividida em duas partes. A primeira apresentada a seguir identifica e conta os sítios com spin -1 cercado por sítios com spin +1 . 
- Para cada sítio $(x, y) \operatorname{com} \sigma=-1$

- Se todos os 8 primeiros vizinhos tem spin $\sigma=+1$

marque o sítio em $(x, y)$ com o valor $i d=-1$

incremente contador.

- Se não

marque o sítio em $(x, y)$ com o valor $i d=0$

- Fim Se

- Fim Para

- $m=5 \cdot$ contador

A segunda parte varre novamente toda a rede e enumera os domínios cristalinos procurando por spins cristalizados com a estrutura apresentada na figura 2.1, apresentada a seguir.

- Para cada sítio $(x, y)$ com $i d=-1$.

- incremente o contador.

- execute recon $(x, y$, contador $)$.

- Fim Para

- $n=$ contador

A função recon é uma função recursiva que varre um único domínio marcando os sítios com o mesmo número $i d=$ contador, seus parâmetros são a posição $(x, y)$ e o contador de domínios, sendo descrita por: 
Função recon $(x, y$, contador $)$

- Se o sítio $(x, y)$ está marcado com $i d=-1$.

- marque o sítio em $(x, y)$ com $i d=$ contador.

- execute recon $(x+2, y+1$, contador $)$.

- execute recon $(x-2, y-1$, contador $)$.

- execute recon $(x+1, y-2$, contador $)$.

- execute recon $(x-1, y+2$, contador $)$.

- execute recon $(x+2, y-1$, contador $)$.

- execute $\operatorname{recon}(x-2, y+1$, contador $)$.

- execute $\operatorname{recon}(x-1, y-2$, contador $)$.

- execute recon $(x+1, y+2$, contador $)$.

- Fim Se

O valor máximo alcançado pela variável contador após a varredura de toda a rede vai ser o número de domínios cristalinos do sistema. Com isso e o número total de sítios cristalizados obtidos na primeira parte, temos diretamente o tamanho médio dos domínios cristalinos dado por

$$
l_{0}=\frac{m}{n} .
$$

\subsection{Regimes}

O tamanho médio dos domínios cristalinos $l_{0}$ e o excesso de energia estão relacionados e esta relação é exata no limite termodinâmico para o estado 


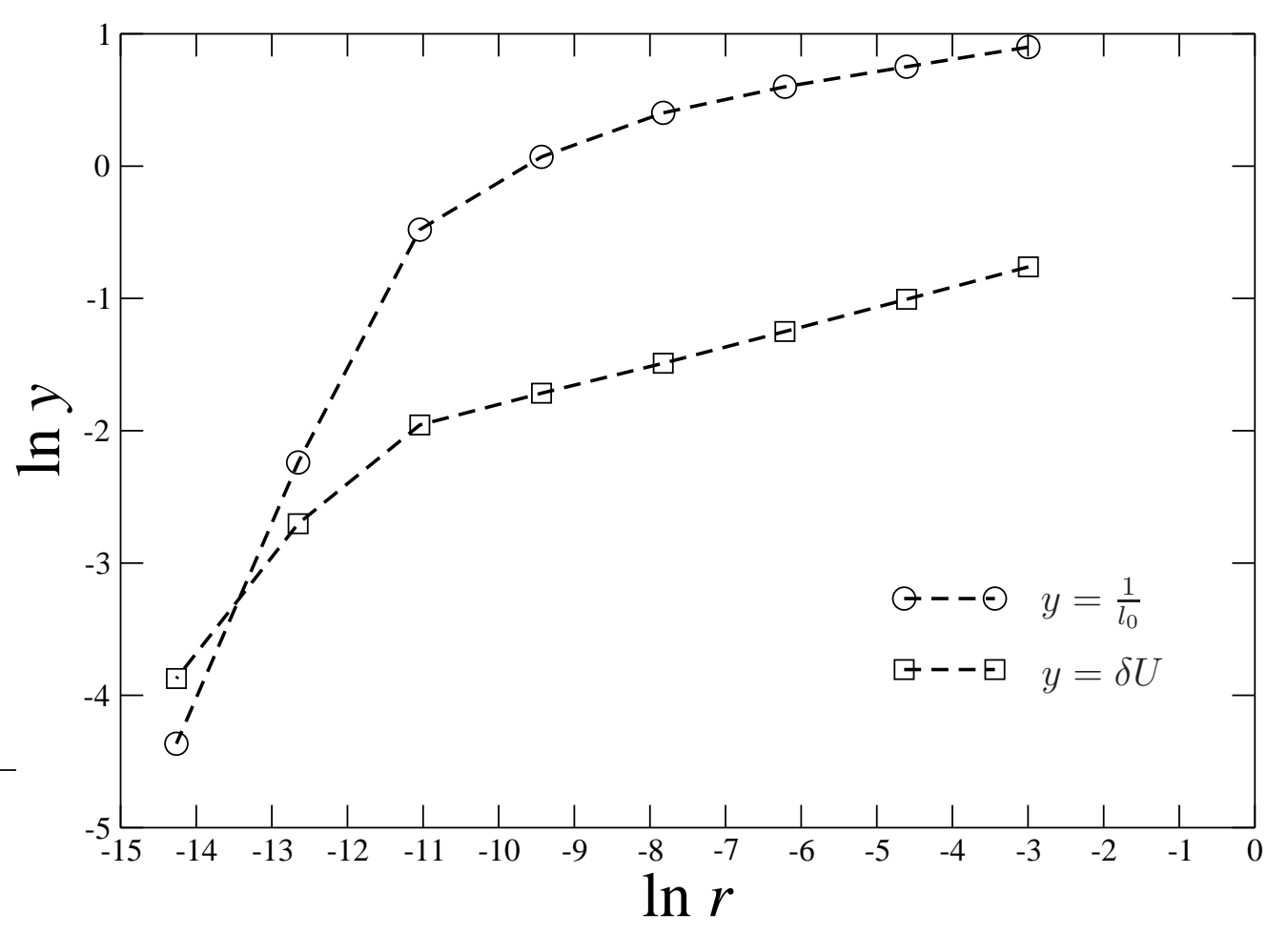

Figura 3.9: Gráfico do logaritmo da diferença da energia a temperatura nula e a energia do estado cristalino pelo logaritmo da velocidade de resfriamento.

fundamental, já que no estado fundamental o excesso de energia é nulo e existe um único domínio cristalino que ocupa o sistema inteiro, ou seja, o tamanho médio dos domínios cristalinos é o tamanho do sistema.

No gráfico da figura 3.9 verificamos a validade de relação 3.3 e vemos que o sistema apresenta pelo menos dois regimes de resfriamento distintos para taxas de resfriamento maiores e menores que aproximadamente $10^{-5}$.

O comportamento do sistema para as taxas de resfriamento maiores sugere que o estado fundamental é inacessível por resfriamento contínuo, pois esta parte da curva, tanto para o excesso de energia como para o tamanho médio dos domínios cristalino, é praticamente uma reta com coeficiente 
angular menor que um.

$$
\begin{aligned}
& \frac{\mathrm{d} \ln l_{0}}{\mathrm{~d} \ln r}<1 \\
& \frac{\mathrm{d} \ln \delta u}{\mathrm{~d} \ln r}<1
\end{aligned}
$$

Vemos que há uma aumento significativo na inclinação da parte lenta das curvas, onde a inclinação do tamanho médio dos domínios cristalinos $l_{0}$ é maior que um e a inclinação do excesso de energia aproximou-se de um, mais ainda é menor que um. Faltam pontos para poder ajustar uma reta adequadamente para concluir se estes dados sugerem ou não que o sistema realmente alcançará o estado fundamental por resfriamento contínuo.

De fato, entre as 197 simulações que fizemos para a taxa de resfriamento $64 \cdot 10^{-8}$ observamos que as configurações resultantes eram a configuração cristalina ou uma configuração policristalina muito semelhante com as configurações apresentadas nas figuras 4.3c e 4.6b. 


\section{Capítulo 4}

\section{Resfriamento súbito}

\subsection{Introdução}

Estudamos o comportamento dinâmico do modelo com resfrimentos súbitos. O sistema é preparado numa configuração aleatória, que corresponde a uma temperatura infinita. Em sequida os spins são atualizados com o algoritmo de Metropolis, isto é, os sítios são aleatoriamente escolhidos e seu spin é trocado com probabilidade $p$ de acordo com o peso de Metropolis dado por

$$
p=\min \left\{1, e^{-\beta \Delta E}\right\} .
$$

Cada atualização completa da rede equivale a um passo de Monte Carlo $p m c$, que é a nossa unidade fundamental de tempo.

Essa simulação imita o contato térmico do sistema com um banho térmico à temperatura $T$. Portanto, nossa simulação imita um resfriamento súbito de uma temperatura infinita para uma temperatura $T$. Dessa forma, observamos 
a maneira na qual o sistema é atraído por mínimos de energia, tanto os locais como os globais. Os mínimos locais são também chamados de bacias de potencial.

\subsection{Simulação}

Para cada passo de Monte Carlo determinamos a energia por sítio para um sistema de tamanho $100 \times 100$ com condições periódicas de contorno. Na figura 4.1 mostramos a energia por spin $u$ versus o tempo $t$, e na figura 4.2 mostramos o tamanho médio dos domínios cristalinos $l$ versus o tempo $t$. Os gráficos são resultados das mesmas simulações e cada curva é a média de 4 a 30 realizações.

Observa-se nestes gráficos que para temperaturas menores que $T=0,1$, o sistema estaciona em patamares de energia próximos de $u=-0,75$, e não observamos no intervalo de duração do experimento sinais claros de mudanças. Para a temperatura nula, a dinâmica de Metropolis só permite a troca de um spin se o sistema diminuir a energia total. Sendo assim, o patamar atingido pelo resfriamento súbito a temperatura de banho térmico nula persistirá eternamente. Este estado que originou esee patamar é um estado metaestável, e para o sistema escapar dessa configuração é preciso acumular um número suficiente de flips que ocorrem com probabilidade $p$ afim de superar a barreira potencial que o separa do próximo estado de menor energia. Na figura 4.3a apresentamos um exemplo dessa configuração metaestável e identificamos este estado como vítreo, por ser um estado metaestável de longa duração e por ser desordenado. 


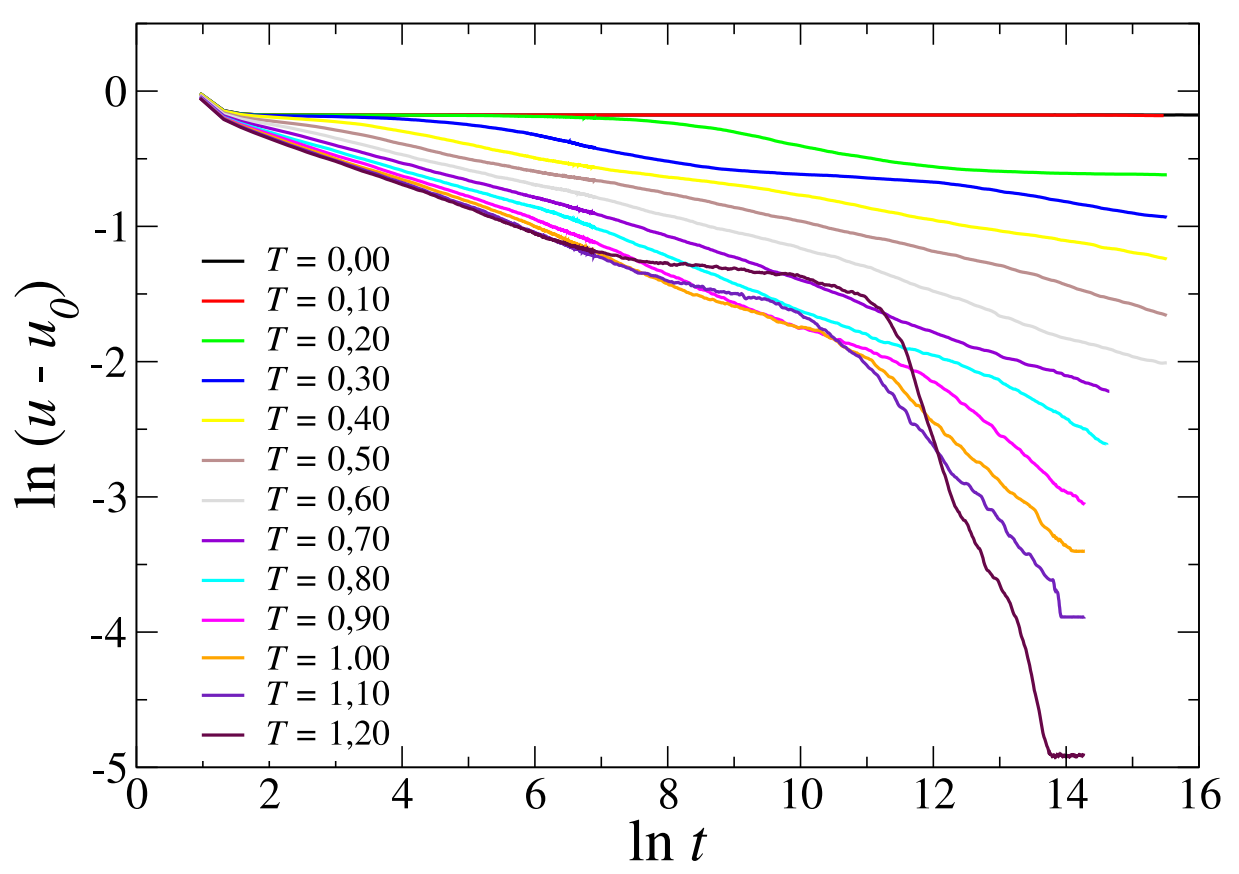

Figura 4.1: Logaritmo do excesso de energia versus o logaritmo do tempo para diversas temperaturas de banho térmico $T$. 


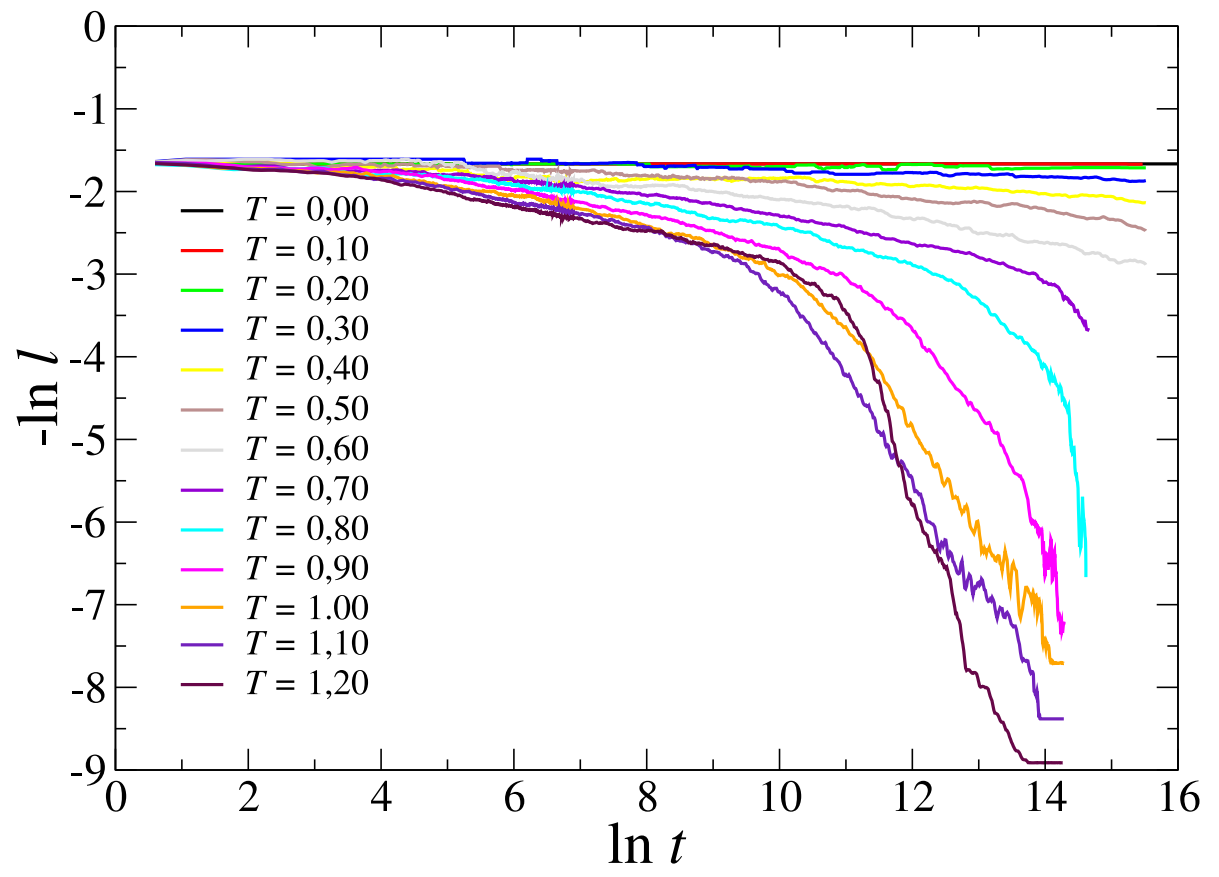

Figura 4.2: Logaritmo do inverso do tamanho médio dos domínios cristalinos $-\ln l$ versus o logaritmo do tempo para diversas temperaturas de banho térmico $T$. 


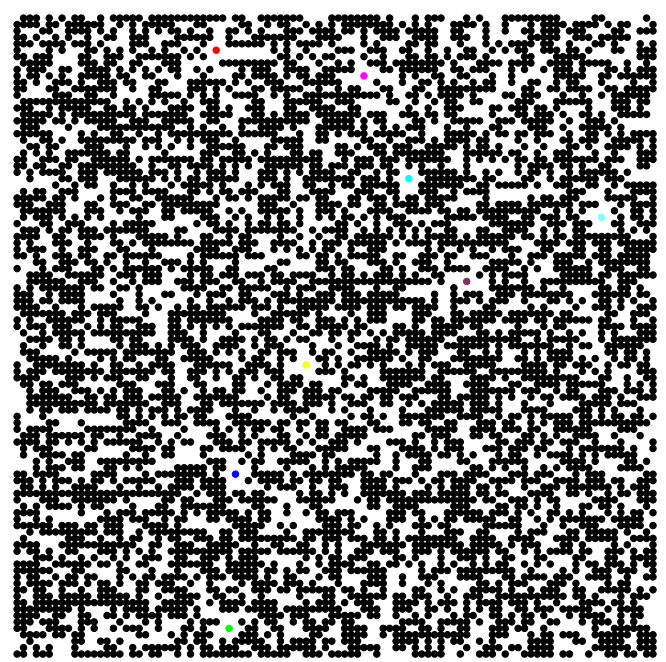

(a) $\quad T=0,0$

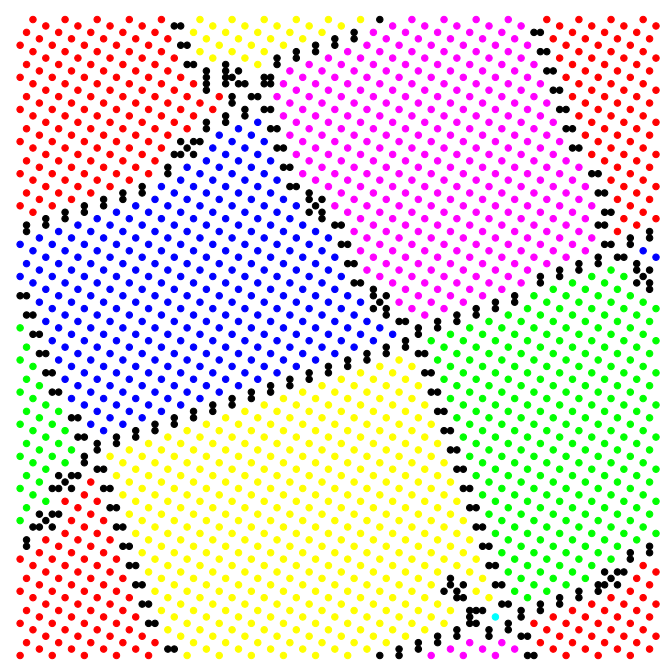

(c) $T=1,2$

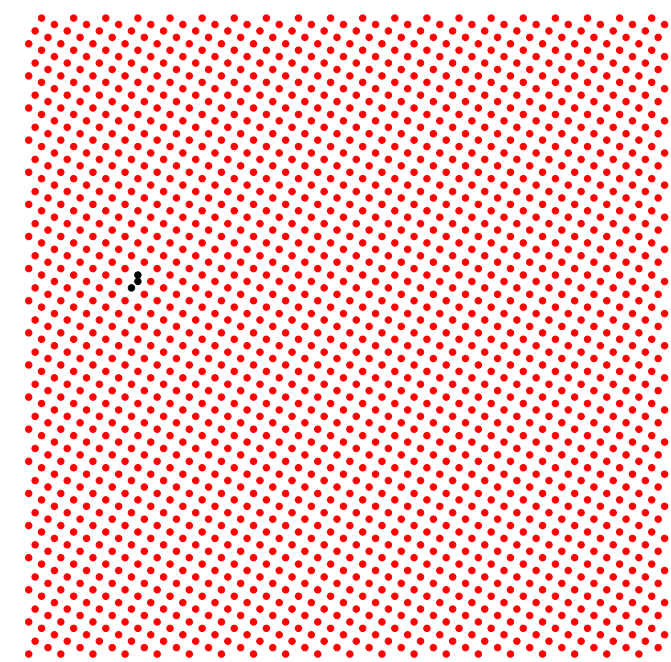

(b) $T=1,1$

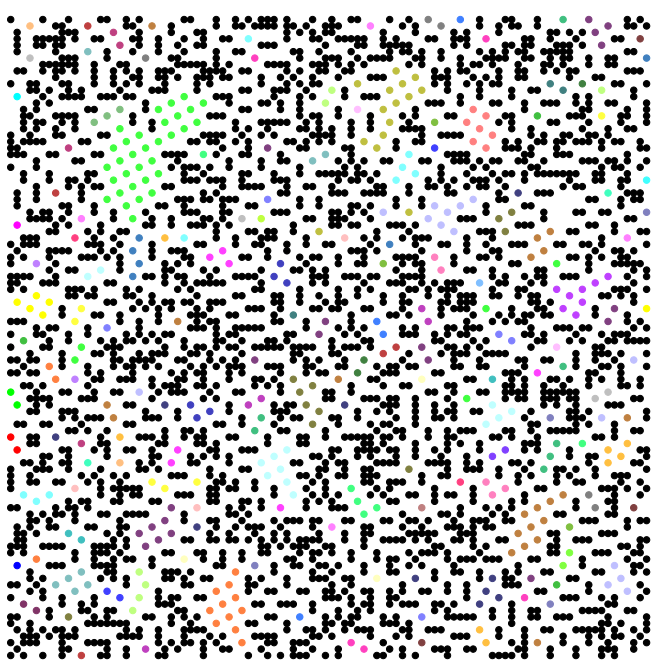

(d) $\quad T=1,3$

Figura 4.3: Exemplo de configuração do sistema após 8462566 passos de Monte Carlo a temperatura de banho térmico nula $T=0,0, T=1,1, T=$ 1,2e $T=1,3$ respectivamente. Foi utilizada a mesma seqüência de números aleatórios para as quatro simulações. (c) A configuração apresentada não é qualitativamente única. Às vezes o sistema alcança o estado fundamental. 


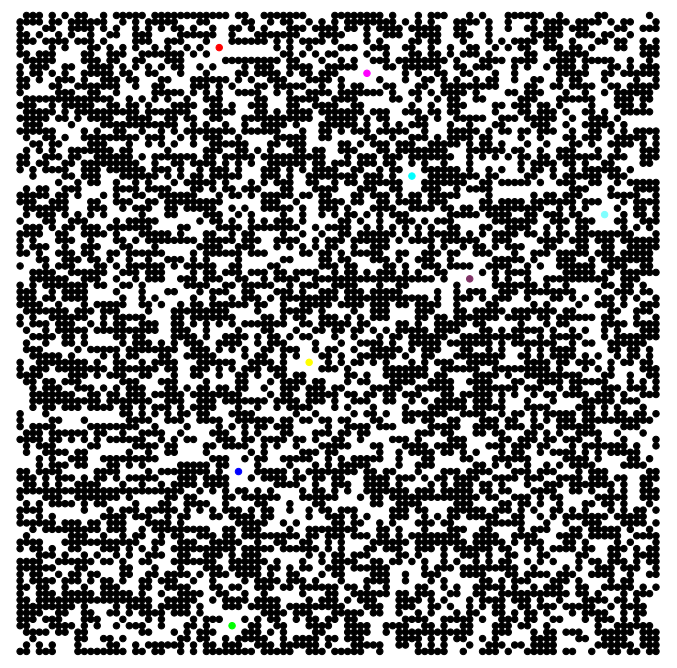

(a) $\quad t=64$

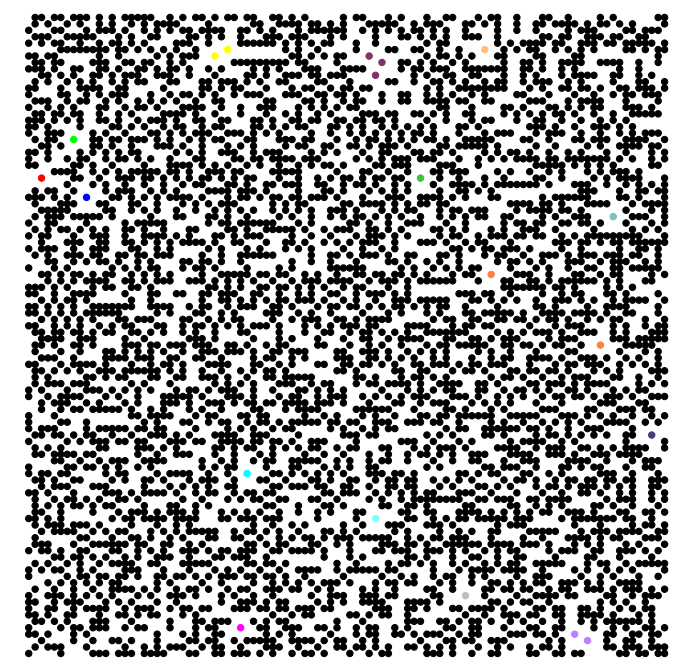

(b) $\quad t=8462566$

Figura 4.4: Fotografias da configuração do sistema no instante $t$, resfriado a temperatura de banho térmico $T=0,2$. Apenas os sítios com spin -1 são apresentados, os sítios pertencentes ao mesmo domínio cristalino são coloridos com a mesma cor. 
Tabela 4.1: Tabela da temperatura de banho térmico $T$ pela inclinação da reta ajustada no final da curva do logaritmo do excesso de energia do resfriamento súbito apresentado na figura 4.1.

\begin{tabular}{|l|r|}
\hline$T$ & tangente \\
\hline 0,0 & $-0,000133$ \\
0,1 & $-0,00299$ \\
0,2 & $-0,00782$ \\
0,3 & $-0,0790$ \\
0,4 & $-0,0843$ \\
0,5 & $-0,146$ \\
0,6 & $-0,147$ \\
0,7 & $-0,154$ \\
0,8 & $-0,253$ \\
0,9 & $-0,414$ \\
1,0 & $-0,461$ \\
1,1 & $-0,566$ \\
1,2 & $-1,118$ \\
\hline
\end{tabular}

Já para temperaturas entre $T=0,2$ e $T=0,5$ observamos a existência de um segundo patamar de energia próximo a $u=-1,08$ e como esperado, observamos que a persitência desses patamares diminui com o aumento da temperatura, sendo sua existência imperceptível a $T=0,6$. Na figura 4.4 apresentamos um exemplo de configuração para cada patamar. As simulações foram realizadas usando deliberadamente a mesma seqüência de números aleatórios, onde destacamos a semelhança nos pequenos domínios cristalinos comuns entre as figuras $4.4 \mathrm{a}$ e $4.3 \mathrm{a}$.

Para temperaturas entre $T=0,6 T=0,9$, o sistema evolui sem apre- 


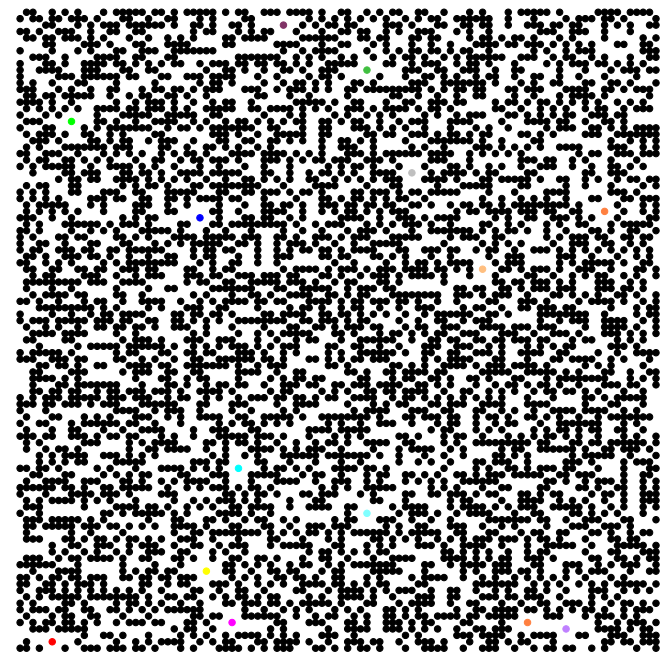

(a) $\quad t=64$

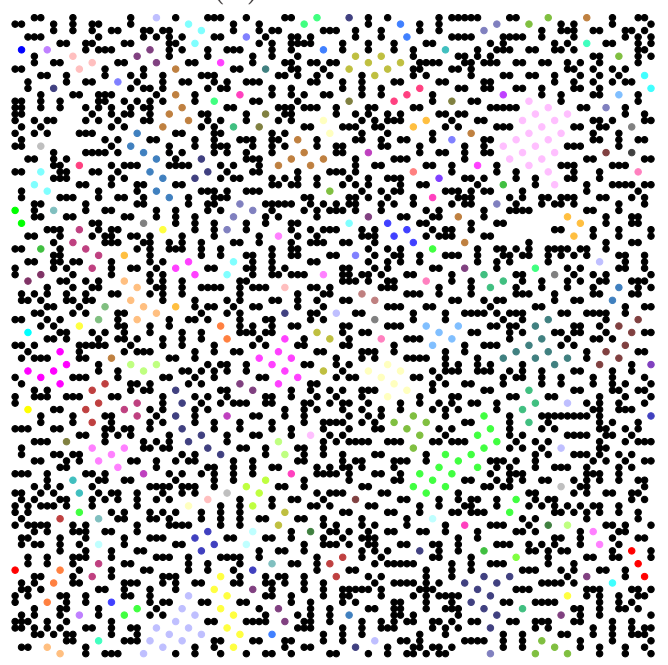

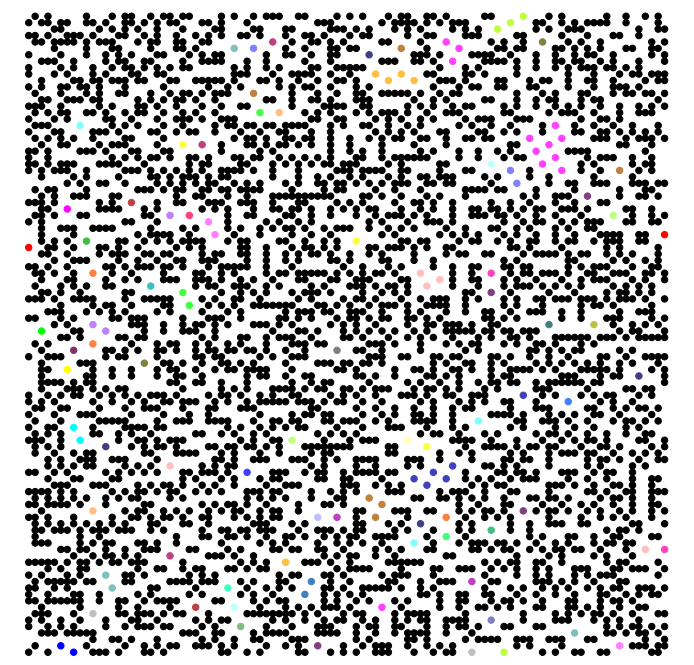

(b) $\quad t=4108$

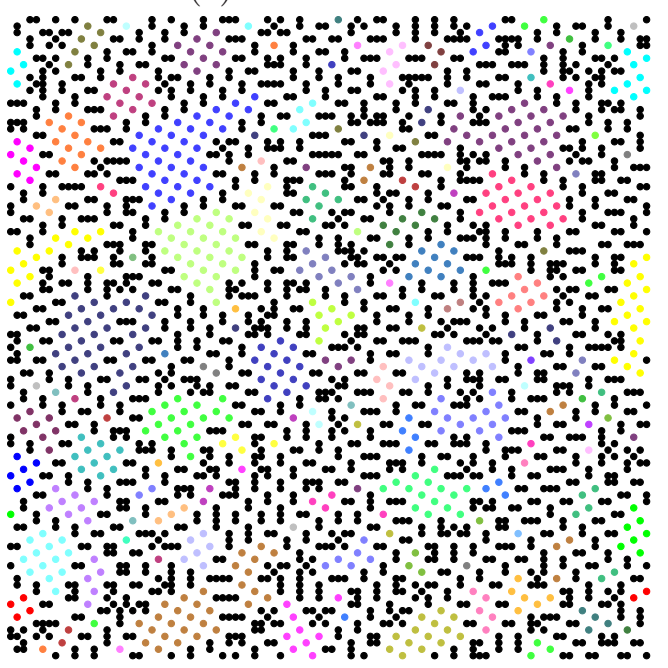

(d) $\quad t=8462566$

Figura 4.5: Fotografias da configuração do sistema no instante $t$, resfriado a temperatura de banho térmico $T=0,6$. Apenas os sítios com spin -1 são apresentados, os sítios pertecentes ao mesmo domínio cristalino são coloridos com a mesma cor. 


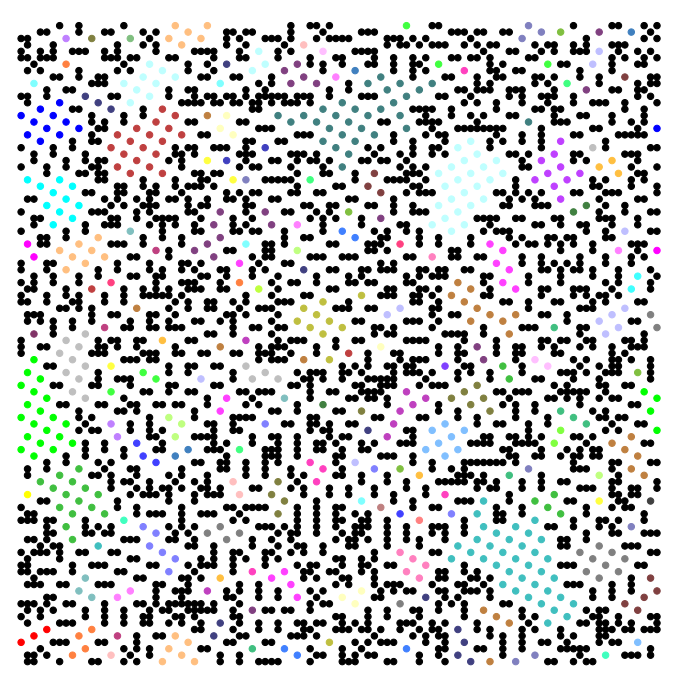

(a) $\quad t=8222$

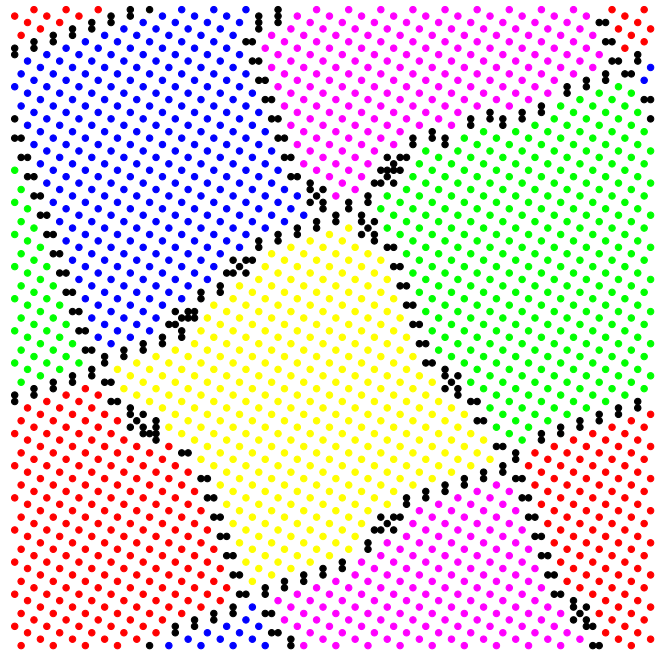

(b) $\quad t=527496$

Figura 4.6: Fotografias da configuração do sistema no instante $t$, resfriado a temperatura de banho térmico $T=1,2$. Apenas os sítios com spin -1 são apresentados, os sítios pertecentes ao mesmo domínio cristalino são coloridos com a mesma cor.

sentar os patamares presentes em temperaturas menores. Isto significa que apesar da existência do estado metaestável, sua influência foi reduzida a tal ponto com o aumento da temperatura que não consegue mais manter estacionado o sistema nos patamares mencionados. Ao mesmo tempo, a influência desses estados metaestáveis e os múltiplos estado fundamentais justifica o modo não exponêncial que o sistema relaxa. Na figura 4.5 apresentamos a configuração do sistema em quatro instantes diferentes.

A temperaturas maiores que $T=1,0$, o sistema começa a sentir a influência do estado líquido e em $T=1,3$ o sistema apresenta o comportamento típico da transição de primeira ordem. O sistema eventualmente evolui para o estado apresentado na figura 4.3c, que é um exemplo de estado policristalino, apesar de ser também um estado metaestável de longa duração, não 
poder ser considerado um estado vítreo. 


\section{Capítulo 5}

\section{Conclusões}

Estudamos o CTLS como um modelo que imita o comportamento dos vidros estruturais, o líquido super-resfriado, o policristal e o cristal. Analisamos o modelo sob ótica do equilíbrio termodinâmico através de aproximações analíticas e simulações de Monte Carlo com dinâmica de Metropolis. Comparamos as aproximações com os resultados das simulações e mostramos que o modelo apresenta a fenomenologia típica da transição de primeira ordem.

Fizemos também investigações fora do equilíbrio com resfriamentos gradativos e resfriamentos súbitos com o propósito de simular o processo de formação de vidros reais.

Nos resfriamentos gradativos constatamos o aparecimento da transição vítrea, estimamos os valores da temperatura de transição vítrea $T_{g}$ e da temperatura de Kauzmann $T_{k}$, e comparamos seu comportamento em função da taxa de resfriamento $r$ com a expectativa experimental.

Nas curvas da energia por spin dos resfriamentos súbitos, observamos o 
sistema relaxar de forma não exponencial e atribuimos a isso a forte influência dos estados metaestáveis. Neste contexto, o estado policristalino e o vítreo têm comportamento semelhante. Entretanto, a metaestabilidade do estado policristalino é claramente conseqüencia da competição entre os múltiplos estados fundamentais. Curiosamente, o modelo apresentou durante o resfriamento súbito, à temperatura de banho térmico nula, estados metaestáveis de duração muito longa com energia maior que a energia do líquido próximo da temperatura de transição líquido-cristal $T_{m}$.

Desenvolvemos um algoritmo rudimentar para caracterizar a cristalização do sistema através da contagem direta da quantidade de sítios que estão no estado cristalino, assim como a quantidade de sítios pertencentes a cada domínio cristalino.

Com esta ferramenta determinanos diretamente o tamanho médio dos domínios cristalinos $l_{0}$, e com este parâmetro discernimos o estado vítreo do estado policristalino que são ambos metaestáveis de longa duração. Verificamos com esta mesma ferramenta que a relação entre o excesso de energia e o tamanho médio dos domínios cristalinos,

$$
\delta u \sim \frac{1}{l_{0}}
$$

nos resfriamentos gradativos é bastante satisfatória para taxas de resfriamento $r$ maiores que $10^{-6}$. 


\section{Referências Bibliográficas}

[1] P. G. Debenedetti and F. H. Stillinger ,Nature 410, 259 (2001).

[2] http://focus.aps.org/story/v15/st20

[3] W. Kauzmann, Chem. Rev. 43, 219 (1948).

[4] Spin Glasses and Random Fields, edited by A. P. Young (World Scientific, Singapore,1998).

[5] A. Lipowski, J. Phys. A 30, 7365 (1997); A. Lipowski and D. Johnston, J. Phys. A 33, 4451 (2000).

[6] M. R. Swift, H. Bokil, R. D. M. Travasso and A. J. Bray, Phys. Rev. B 62, $11494(2000)$.

[7] M. J. de Oliveira, A. Petri e T. Tomé, Europhys. Lett. 6520 (2004).

[8] M. J. de Oliveira, A. Petri e T. Tomé, Physica A 342 (1-2), 97-103 (2004).

[9] A. Petri, Braz. J. Phys. 33521 (2003).

[10] M. I. de Berganza, V. Loreto e A. Petri, http://arxiv.org/abs/0706.3534v1 (2007) 
[11] M. I. de Berganza, V. Loreto e A. Petri, http://arxiv.org/abs/cond-mat/0606282v2 (2006)

[12] M. I. de Berganza, E. E. Ferrero, S. A. Cannas, V. Loreto e A. Petri, http://arxiv.org/abs/cond-mat/0610039v1 (2006)

[13] E. E. Ferrero e S. A. Cannas, http://arxiv.org/abs/0707.0265v1 (2007)

[14] A. Cavagna, I. Giardina e T. S. Grigera, J. Chem. Phys. 118, 6974 (2003).

[15] T. S. Grigera e N. E. Israeloff, Philos. Mag. B 82, 313 (2003).

[16] Sílvio R. A. Salinas, Introdução à Física Estatística (EDUSP, São Paulo, 1999).

[17] M. Plischke e B. Bergersen, Equilibrium Statistical Physics (World Scientific, Canada, 1999), 2a ed.

[18] T. Tomé e M. J. de Oliveira, Dinâmica Estocástica e Irreversibilidade (EDUSP, São Paulo, 2001).

[19] W. H. Press, S. A. Teukolsky, W. T. Vetterling e B. P. Flannery, Numerical Recipes in C (Cambridge, Reino Unido, 1997).

[20] D. P. Landau e K. Binder, A Guide to Monte Carlo Simulations in Statistical Physics (Cambridge, Reino Unido, 2000).

[21] M. Lüscher, Comput. Phys. Commun. 79, 100 (1994).

[22] http://www.gnu.org/software/gsl/

[23] http://gcc.gnu.org/ 
[24] Ernst-Joachim Donth, The glass transition: relaxation dynamics in liquids and disordered materials (Springer, Berlin, 2001). 